Planetary Protection Issues in the Human Exploration of Mars

Final Report May 9, 2005

\author{
Editors: M. E. Criswell \\ M. S. Race \\ J. D. Rummel \\ A. Baker
}

Report of a Workshop held June 20-23, 2001 at

Pingree Park Mountain Campus Colorado State University Fort Collins, Colorado

Workshop sponsored by NASA Ames Research Center Moffett Field, CA 94035 
Since its founding, NASA has been dedicated to the advancement of aeronautics and space science. The NASA Scientific and Technical Information (STI) Program Office plays a key part in helping NASA maintain this important role.

The NASA STI Program Office is operated by Langley Research Center, the Lead Center for NASA's scientific and technical information. The NASA STI Program Office provides access to the NASA STI Database, the largest collection of aeronautical and space science STI in the world. The Program Office is also NASA's institutional mechanism for disseminating the results of its research and development activities. These results are published by NASA in the NASA STI Report Series, which includes the following report types:

- TECHNICAL PUBLICATION. Reports of completed research or a major significant phase of research that present the results of NASA programs and include extensive data or theoretical analysis. Includes compilations of significant scientific and technical data and information deemed to be of continuing reference value. NASA's counterpart of peer-reviewed formal professional papers but has less stringent limitations on manuscript length and extent of graphic presentations.

- TECHNICAL MEMORANDUM. Scientific and technical findings that are preliminary or of specialized interest, e.g., quick release reports, working papers, and bibliographies that contain minimal annotation. Does not contain extensive analysis.

- CONTRACTOR REPORT. Scientific and technical findings by NASA-sponsored contractors and grantees.
- CONFERENCE PUBLICATION. Collected papers from scientific and technical conferences, symposia, seminars, or other meetings sponsored or cosponsored by NASA.

- SPECIAL PUBLICATION. Scientific, technical, or historical information from NASA programs, projects, and missions, often concerned with subjects having substantial public interest.

- TECHNICAL TRANSLATION. Englishlanguage translations of foreign scientific and technical material pertinent to NASA's mission.

Specialized services that complement the STI Program Office's diverse offerings include creating custom thesauri, building customized databases, organizing and publishing research results ... even providing videos.

For more information about the NASA STI Program Office, see the following:

- Access the NASA STI Program Home Page at http://www.sti.nasa.gov

- E-mail your question via the Internet to help@ sti.nasa.gov

- Fax your question to the NASA Access Help Desk at (301) 621-0134

- Telephone the NASA Access Help Desk at (301) 621-0390

- Write to:

NASA Access Help Desk

NASA Center for AeroSpace Information 7121 Standard Drive Hanover, MD 21076-1320 


\title{
Planetary Protection Issues in the Human Exploration of Mars
}

Final Report May 9, 2005

\author{
Editors: M. E. Criswell \\ M. S. Race \\ J. D. Rummel \\ A. Baker
}

Report of a Workshop held June 20-23, 2001 at

Pingree Park Mountain Campus Colorado State University Fort Collins, Colorado

Workshop sponsored by NASA Ames Research Center Moffett Field, CA 94035

National Aeronautics and

Space Administration

Ames Research Center

Moffett Field, California 94035-1000 


\section{Acknowledgments}

The preliminary goals, objectives, and format of this Workshop resulted largely from discussions among John Rummel, Gary Coulter, and Marvin Criswell. A Workshop Coordinating Committee comprised of Chris McKay and Mike Duke, along with Marvin Criswell as the Workshop host, worked with John Rummel in formulating the Workshop organization and invitation list. Financial support for the Workshop was provided by NASA Ames Research Center, with further project guidance provided by Donald DeVincenzi, Chief, Space Science Division, at NASA Ames.

Chris McKay's effective leadership and technical advice, along with John Rummel's many contributions, were critical to the success of the Workshop. Although scheduling conflicts kept several key individuals involved in the Workshop planning from attending the actual Workshop (Michael Duke, Donald DeVincenzi, and Gary Coulter), their contributions to the overall Workshop effort should be noted. When assembled, the remainder of the Workshop participants represented a wealth of professional knowledge and interest in the planetary protection concerns of future space endeavors. Their participation formed the core of the Workshop and made it successful. The roles and contributions of the Working Group rapporteurs and recorders were essential to the Workshop, and their reports form a vital resource for these proceedings.

In addition to recorded notes by each working group, other input from Workshop participants, including the students in attendance, were very helpful in compiling this report. Special thanks go to Lee Criswell, graduate student and wife of workshop host, and $1^{\text {st }}$ editor Marvin Criswell. Her background in life sciences was helpful on many of the biological and health related topics both during the workshop and afterwards. Margaret Race's review and organization of technical content was greatly appreciated during the process of preparing this report. Additional review comments and suggestions from several others, most notably Sara Acevedo and Gary Coulter, also were very helpful in its preparation.

Marvin Criswell

Fort Collins, Colorado

July 2004

Available from:

NASA Center for AeroSpace Information 7121 Standard Drive

Hanover, MD 21076-1320

(301) 621-0390
National Technical Information Service 5285 Port Royal Road Springfield, VA 22161

(703) 487-4650 


\section{Table of Contents}

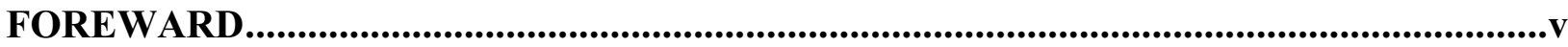

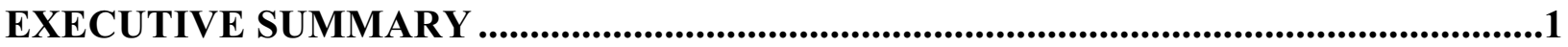

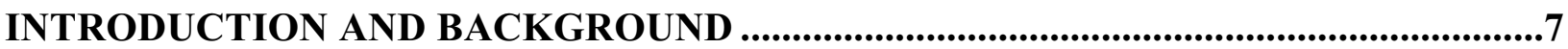

ORGANIZATION OF THE WORKSHOP .............................................................................11

OVERVIEW COMMENTS ON THE GENERAL WORKSHOP TOPICS

(MARVIN CRISWELL) ..............................................................................................................15

WORKING GROUP REPORTS........................................................................................19

Working Group WG1 - Protecting Mars and Science: "Protecting Mars and Mars Samples from

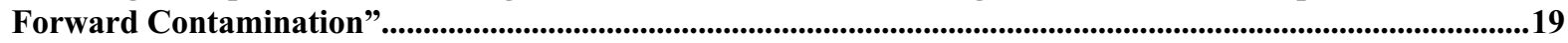

Working Group WG2 - Protecting Human Health: "Protecting Human Health Against Risks of

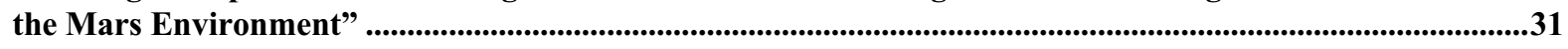

Working Group WG3: Protecting Earth: “Preventing Back Contamination of Earth from Mars Return".

Workshop Group WG4A and WG4B - Operations I and Operations II: “Enabling a Safe, Productive Human Presence in the Exploration of Mars" ..........................................................................................41

Workshop Group WG4B - Operations II: "Enabling a Safe, Productive Human Presence in the Exploration of Mars"

PLENARY DISCUSSIONS AND RECOMMENDATIONS ON OPERATIONS...............57

OVERALL WORKSHOP CONCLUSIONS AND RECOMMENDATIONS......................61

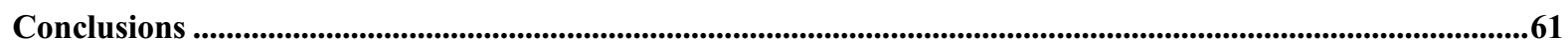

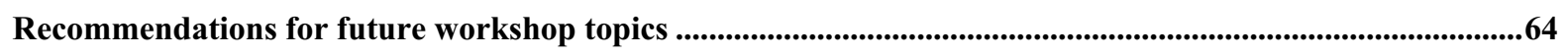

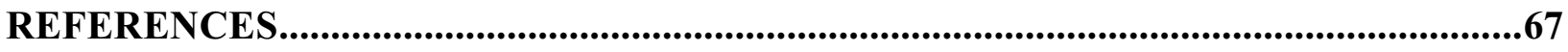

APPENDIX A: WORKSHOP VENUE AND AGENDA ............................................69

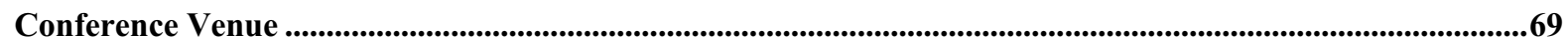

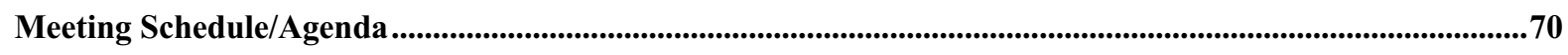


APPENDIX B: WORKING GROUP COMMITTEE MEMBERS.......................................73

APPENDIX C: WORKSHOP PARTICIPANTS LIST AND ADDRESSES ......................75 


\section{Foreword}

This workshop report, long delayed, is the first $21^{\text {st }}$ century contribution to what will likely be a series of reports examining the effects of human exploration on the overall scientific study of Mars. The considerations of human-associated microbial contamination were last studied in a 1990 workshop ("Planetary Protection Issues and Future Mars Missions," NASA CP-10086, 1991), but the timing of that workshop allowed neither a careful examination of the full range of issues, nor an appreciation for the Mars that has been revealed by the Mars Global Surveyor and Mars Pathfinder missions. Future workshops will also have the advantage of Mars Odyssey, the Mars Exploration Rover missions, and ESA's Mars Express, but the Pingree Park workshop reported here had both the NRC's (1992) concern that "Missions carrying humans to Mars will contaminate the planet" and over a decade of careful study of human exploration objectives to guide them - and to reconcile. A daunting challenge, and one that is not going to be simple (as the working title of this meeting, "When Ecologies Collide?" might suggest), but it is clear that planetary protection issues will have to be addressed to enable human explorers to safely and competently extend out knowledge about Mars, and its potential as a home for life-whether martian or human.

John D. Rummel

Planetary Protection Officer

NASA Headquarters

May 2005 


\section{Planetary Protection Issues In the Human Exploration of Mars}

\section{Executive Summary}

The Workshop on "Planetary Protection Issues in the Human Exploration of Mars" focused on the human exploration of Mars from a planetary protection perspective. The introduction of astronauts, with all their needs and abilities, into the exploration mission greatly expands both the mission's scope and the planetary protection concerns compared to those of robotic-sampling and other precursor missions without humans. The initial working title of the meeting was "When Ecologies Collide? Planetary Protection Issues in the Human Exploration of Mars." The question mark after "When Ecologies Collide?" was deliberate, as we do not yet know if there is any life and ecology on Mars other than our own. Thus, the ecologies of concern during human missions are those of Earth, possibly that of Mars, and potentially an ecology resulting from the interaction of the two.

With its primary focus on future human exploration of Mars, the main question for the Workshop was: Can human exploration of the martian surface be done effectively and without harmful contamination? The many concerns with respect to contamination can be placed within three general categories: (1) protecting Mars and Mars samples from forward contamination, i.e., "Protecting Mars and Science," (2) protecting astronaut health against risks from the Mars environment, i.e., "Protecting Human Health," and (3) preventing back contamination of Earth from possible Mars contaminant sources, i.e., "Protecting Earth." Inherent in the question of "can it be done?" is an examination of key human and project needs and critical mission operations that raise planetary protection concerns, along with strategies to mitigate or negate these concerns. As in robotic exploration missions, assuring that Mars samples are free from contamination is a critical science issue, regardless which stage the contamination occurs - from sampling through evaluation on site or after sample return. Human missions to Mars entail all the issues already examined for robotic missions, in addition to others arising through the direct involvement of humans and their accompanying microbial companions. Although these complexities have been previously identified and discussed, this is the first workshop specifically devoted to this topic in more than a decade. As a result, much of its thrust was exploratory in nature.

The Workshop brought together a multitalented group of 29 professionals from various academic institutions, NASA locations, industry, and other organizations - plus several students - to explore the key issues and document their findings. Included areas of expertise ranged from the basic sciences and astrobiology through life support and space medicine, to operational areas such as mission planning and support. This 2-1/2 day Workshop was held at conference facilities on the Pingree Park mountain campus of Colorado State University, located northwest of Fort Collins, Colorado.

The Workshop was organized to address the critical issues associated with human exploration of Mars by examining the following topics:

1. Review of current human capabilities and plans for the scientific exploration of Mars;

2. Protecting Mars and science (Mars samples) from forward contamination, i.e., Earth to Mars;

3. Protecting human health against risks from the Mars environment; 
4. Preventing back contamination of Earth from returned martian samples, astronauts and mission elements; and

5. Operations enabling a safe, productive human presence in the exploration of Mars.

After an initial series of presentations on background topics about the martian environment, human operations in space, astrobiology, terrestrial extremophiles, and general planetary protection concerns, the Workshop participants were assigned to one of the following three working groups to explore, discuss and later report back to the Workshop in plenary session:

WG1 - Protecting Mars and Mars Sampling from Contamination - "Protecting Mars \& Science" WG2 - Protecting Astronauts against Risks of the Mars Environment - "Protecting Human Health" WG3 - Preventing Back Contamination of Earth from Mars Return - "Protecting Earth”

After presentation and discussion of each of the topics in plenary session, participants were subsequently reassigned to one of two parallel working groups emphasizing mission operations and humans working on Mars:

WG4A - Enabling a Safe, Productive Human Presence in Mars Exploration - “Operations I" WG4B - Same topic - “Operations II”

Each of these parallel "Operations" working groups was instructed to examine and report back on six scenarios with regard to planetary protection: (1) distant surface sample collection; (2) sample analysis; (3) in situ resource utilization (ISRU) at a martian base; (4) plant growth experiments and greenhouses supplying food; (5) sub-surface sampling (to $10 \mathrm{~m}$ and to $1 \mathrm{~km}$ depth); and (6) what to do if and when life is found.

In keeping with the Workshop's exploratory nature, a wide range of topics and questions was discussed. Questions included the uncertainties of human Mars mission design, available technologies at the time of a mission, mission tasks, and the overriding issue of "is there life on Mars?" If so, where is it found and what is it like? Is it or other aspects of the martian environment dangerous to the astronauts and to Earth?

Some of the Workshop attendees had participated in previous NASA workshops on the development of a protocol for handling sample returned from Mars during future robotic missions. This led to a general awareness of overall planetary protection procedures and concerns, and contributed useful information to discussions about humans on Mars.

In the Workshop discussions, questions of overall mission philosophy and approach often arose. It was recognized that for human missions (like robotic ones) the landing site selection and exploration options that hold the greatest scientific promise are also likely to be those with the highest risks regarding planetary protection. Two of the three primary working groups independently explored a strategy that included a scenario with the initial human base located on a site that was well defined by precursor mission information. Presumably, this would be a site of low environmental risks, but in the proximity of more interesting martian features. Validation of conditions at the base site would be followed by exploration forays, first using robots, and later, combinations of robotics and astronaut explorers in closed rovers. Forays would have the primary task of mapping local areas based on environmental risk, with subsequent local surface operations dependent upon these evaluations. 
Workshop Conclusions and General Recommendations. A summary of the Workshop findings ${ }^{1}$ includes a mix of conclusions and recommendations, including:

1. Planetary protection must be assured before any contact is made with martian materials. Thus, planetary protection considerations must be included as a part of the initial stages of mission and hardware design for interplanetary missions, especially for, but not limited to, surface exploration missions that include humans.

2. Including humans in martian missions significantly increases the challenges for planetary protection assurance because humans are hosts to a complex endogenous microbial community related to digestion and other bodily functions. Unlike robotic missions, all components of a human mission cannot be sterilized before launch, resulting in the many planetary protection issues and risks that arise with the addition of a human component to the mission.

3. Humans on a future Mars mission will have unique capabilities in recognizing likely sites and specific local physical features that might harbor life or otherwise be of very high interest. Their insight will be useful for deciding where to sample and providing ongoing evaluations of exploration conditions.

4. All operations of an initial human mission to Mars should include isolation of humans from any direct contact with materials from Mars for planetary protection purposes.

5. The planning of human missions, including base site location and mission objectives, should follow from precursor robotic information and evaluations at local sites, as well as information form sample return missions.

6. Further definition is needed for a system describing and categorizing martian sites of special scientific interest and of level of contamination concern. These classification systems should be developed and employed in future planetary protection protocols, as well as in operational plans for later human missions to Mars.

7. Further study must be given to long-term forward planetary protection concerns and the possible interaction of any forward contaminants with surface features and disturbances on Mars given that it will be difficult to guarantee all human life processes and mission operations are conducted within entirely closed systems. There may be critical emerging issues regarding possible ecological developments on Mars that are associated with the possible survival and evolution of Earth organisms in the presence of martian materials.

8. At present, it is not easy to apply definitions of Earth life to possible martian life. Life on Earth has been found in locations with temperature, pressure, $\mathrm{pH}$, radiation, or other extreme physical or chemical conditions previously thought to be incompatible with life. It is possible that some martian life may likewise exist in forms and in locations we cannot now fully predict.

9. Additional development and design attention will be needed for many exploration, sampling, and base activities to assure both effective operation and the required level of planetary protection assurance. Based on current and anticipated technologies, subsurface sampling operations appear particularly problematic for use on Mars with respect to planetary protection issues.

\footnotetext{
${ }^{1}$ A summary paper on the findings of this workshop was published earlier, as well (Race et al., 2003)
} 
10. General human factors need to be considered along with planetary protection issues for a human mission to Mars. Physical effects that may lead to debilitation and reduced performance in astronauts could also lead to unintended actions, and in turn, to mishaps with potentially serious planetary protection consequences. Mistakes are much more likely when people are tired, ill, and/or stressed or overly stressed.

11. The first human mission to Mars will face a significant level of risk, as it will involve complex transportation, life support and base systems; long mission times (both transfer and on-surface times) governed by orbital mechanics; and many unknowns. Regardless of prior testing and simulations, the first human mission will be the initial test of the full system. Planetary protection risks are among many risks to be identified and evaluated together, then reduced, mitigated, or eliminated when possible to help assure mission success.

12. The Workshop generally concluded that it is conceptually possible to develop systems, exploratory approaches, and operations plans to enable a safe, productive human mission to the Mars surface. Although planetary protection requirements are typically compatible with good science practices and prudent engineering designs and mission architecture, these requirements will certainly affect the design, operations, and costs for long duration human missions.

Needed Areas of Research - The conclusions and general recommendations presented above identify many areas where a better knowledge base and/or improved technology are needed prior to a human mission to Mars. Among the many areas in need of additional research, the following were specifically noted:

1. Define the spatial dispersion of dust and contaminants on Mars by wind and other means.

2. Describe the potential impacts of each of the many human support activities expected in the operation of a human-occupied martian base, e.g., breathing oxygen, food supply, waste management, etc.

3. Determine how robotics can best help conduct operations on Mars in a way consistent with planetary protection concerns, both independently during precursor missions and in conjunction with humans in later missions.

4. Improve space suit designs consistent with planetary protection needs, especially for the demands of human activities on the martian surface located away from pressurized habitats and rovers.

5. Develop technology required for life detection and potential pathogen detection, with a focus on sensitivity and specificity of tests needed to answer the questions of "how clean is clean enough?" and "how 'alive' is indeed alive?"

6. Formulate a site classification systems and biological plausibility map of the martian surface and subsurface based on remote sensing data.

Recommendations for Future Workshops and Workshop Topics - Given the exploratory and general nature of the Workshop, it was not surprising that a myriad of potential future workshop suggestions arose. Obviously, improved information and planning in many areas will be needed before the formulation of detailed human mission protocols, specific tasks and mission operations. In the meantime, participants identified the following potential workshop topics to explore important broad issues with implications for human missions: 
1. "If Life, Then What?" The most mentioned candidate for a future, far-reaching workshop was the topic of "If Life, Then What?," which would address appropriate responses to the discovery of martian life, whether in a robotic mission sample return or during the first or later human mission to Mars.

2. Human Factors: Another suggested workshop would focus on general human health issues, life support, work environment, psychological and other human factors, along with their interaction with planetary protection issues and general performance within an extended mission of many months to a few years.

3, 4. Public Communication: Finally, two additional potential workshop topics were suggested that relate to communications with the public, one addressing general public response to the detection of life on Mars (or elsewhere away from Earth), the other on preparing the public for the possible discovery of non-terrestrial life. 


\section{Introduction and Background}

Sometime within the first several decades of this $21^{\text {st }}$ century, humans will likely venture to Mars, back to the Moon, and possibly to other locations within our solar system. Their tasks will be first to explore these "new worlds," later to utilize the resources of these planetary surfaces, and even later, perhaps to colonize these sites. Prior to any human missions to Mars, robotic probes will be sent to explore selected locations of the "Red Planet" in detail and return samples to Earth. Information from these precursor missions will be helpful in selecting sites and defining mission constraints and opportunities for human exploration ventures. Discussion and planning for these future human missions have already begun, although they are still in the formative stages. There are many reasons to embark on these ventures, including human curiosity and urge to explore, the quest for information and knowledge, a search for resources, the benefits of international cooperation needed for such notable projects, and the search for a possible future alternate home for the humanity. Earth may not be the only location in the Solar System supporting life at present, and in future generations, humans may indeed inhabit both Earth and beyond.

Both credible science and Hollywood movies warn us that ventures into the unknown involve dangers as well as opportunities. A finite chance exists that these new extraterrestrial worlds may contain substances and perhaps even life forms that are not compatible with human health and the environment of Earth. Similarly, life from Earth, perhaps in the form of bacteria or other microbes that inevitably accompany humans and their wastes, may prove to be contaminants to other sites if any survive and prosper in these new environments free from their natural competitors. In space missions, we must be careful of what we bring with us, what we leave behind, what human explorers are exposed to in these strange new worlds, and especially what is brought back to Earth and how it is subsequently handled. A space exploration policy ignoring these possibilities would entail gambling with the health of our home planet and its inhabitants, as well as other solar system environments. Yet, a policy accepting absolutely no risks may preclude any meaningful exploration efforts. Thus, the priority tasks in our future human adventures beyond Earth are to understand better the possibilities and risks involved; to eliminate, mitigate, and reduce these risks; to plan mission activities that avoid unnecessary exposures; to provide robust operating procedures that are tolerant of unknowns and potential human errors; and to formulate informed policies that are both prudent and permissive of these future human endeavors.

This workshop originally entitled "When Ecologies Collide? Planetary Protection Issues in the Human Exploration of Mars" focused on the human exploration of Mars from a planetary protection standpoint. The inclusion of the question mark in the initial phrase "When Ecologies Collide?" was intentional and serves to emphasize that we do not yet know if there is any life other than our own and thus whether there are any other ecologies in our celestial neighborhood. If there are, future human ventures to sites of these other ecologies may bring Earth life forms into contact with other indigenous ecologies, with unknown consequences of this "collision.", Given the focus of this workshop on future human exploration of Mars, the primary question is: Can human exploration of the martian surface be done effectively and without harmful contamination? The three areas of concern are (1) protecting Mars and Mars samples from forward contamination-"Protecting Mars and Science," (2) protecting astronauts against risks from the Mars environment- "Protecting Human Health," and (3) preventing back contamination of Earth from Mars return-"Protecting Earth."

\footnotetext{
${ }^{2}$ The initial working title of the Workshop was "When Ecologies Collide? Planetary Protection Issues in the Human Exploration of Mars." The introductory phrase has been dropped from the report title. However, the notion of multiple ecologies - those of Earth, possibly Mars, and potentially from an interaction of Earth and martian resources - was a background consideration in all discussions.
} 
Although this three-part organization emphasized the protection of Mars, astronauts, and Earth, the importance of avoiding the contamination of martian sample materials was also considered.

Contamination of Mars samples at any stage-from acquisition through in situ evaluation, or after sample return to Earth—could result in loss of critical scientific information, such as the detection of life.

Contaminants that represent "false positive" indications of martian life could greatly delay or misdirect subsequent exploration efforts. The same types of "forward contamination" mechanisms that could lead to the possible contamination of the Mars environment (e.g., accidental release of human wastes, venting of suits or base habitats, etc.) are also those that could result in the contamination of samples taken during the initial human mission. In Workshop discussions, the focus on "Protecting Mars and Science" actually encompassed the multiple concerns of protecting Mars for the sake of any martian ecology, protecting the integrity of samples taken during initial human missions, and keeping the Mars environment pristine for later science inquiries.

Although not discussed explicitly at the Workshop, the relevant international laws and space policy documents related to planetary protection were used as baseline assumptions during all deliberations. It was noted that the Outer Space Treaty of 1967 (United Nations, 1967) and COSPAR ${ }^{3}$ international policies require that space exploration missions should be accomplished in ways that avoid harmful contamination of the planets and other celestial bodies, and that protect the Earth's biosphere.

Many complications and questions arise when humans are introduced into Mars exploration scenarios. Unlike space hardware, humans cannot be sterilized and the astronauts will be accompanied by the microbial companions in their digestive tract and elsewhere. Rather than the brief "backpacking" Apollo missions to the Moon of only few-days duration, the rules of orbital mechanics dictate that even the initial human mission to Mars could be on the planet's surface or in Mars orbit for a relatively extended period (a range of 30 to 550 days, depending upon the transit path selected). An early return following unexpected mission events is either not possible or would require very large amounts of propulsion fuel. With transit times lasting many months each way, total mission time away from Earth could be as much as two to three years ${ }^{4}$. This is long enough that controlling possible contamination caused through human nutrition, respiration, and digestive wastes is much more challenging than it would be for a weeklong mission to the Moon. The long duration of the martian mission also introduces a greater need for operations such as recycling and on-site plant growth/food production. While these processes can greatly reduce the magnitude of mission expendables at launch, their contamination potential is both considerable and unavoidable.

This Workshop is viewed as an early step in the consideration of planetary protection requirements that will affect both future human missions and precursor Mars robotic efforts, including sample return. In 1992, the National Research Council (NRC, 1992) recommended that human missions to Mars should not be attempted until robotic missions can address the question of life on Mars and gather important

\footnotetext{
${ }^{3}$ COSPAR is the Committee on Space Research, established by the International Council of Scientific Unions (ICSU) in 1958. COSPAR is designated as the international scientific body that coordinates worldwide space research, maintains and promulgates planetary protection policy, and provides guidelines for compliance with the U.N. Outer Space Treaty of 1967.

${ }^{4}$ At the workshop, no specific mission architecture or scenario was used to guide discussions, although it was assumed that human missions would be of significant duration, with perhaps as much as 500-600 days on the martian surface [see: The Mars Surface Mission: A Description of Human and Robotic Surface Activities, 2001, (NASA TP-2001-20937), NASA, Washington, DC.]
} 
precursor science information. Already, plans are underway to develop a comprehensive sample handling protocol for a Mars sample return mission. ${ }^{5}$ Precursor robotic missions may provide only partial answers about possible life on Mars because of limited ability to explore autonomously. The best location for life to exist on Mars is probably well beneath the surface, and at other locations that are generally inaccessible to envisioned robotic spacecraft, robots, and rovers. This Workshop may be an important preliminary step towards eventual development of requirements for the combined robotic and human-aided exploration of Mars.

Eventually, NASA will need to develop protocols for missions traveling to sites that might host nonterrestrial life and determine how to proceed if life is detected under a variety of scenarios. In addition to possible robotic discovery of extraterrestrial life, another credible scenario is one in which life may be detected by humans while exploring on Mars. Accordingly, another motive for the Workshop was to provide mission planners and the NASA Planetary Protection Office with more information and collective knowledge about the circumstances in which humans might find such life and how to determine unequivocally whether a suspected foreign life form is indeed different from Earth life, rather than being a contaminant. Even though discussions of various scenarios were necessarily exploratory and general in nature, they are likely to aid the NASA Planetary Protection Office and the Astrobiology Program in later development of protocols and requirements to understand and prevent inadvertent biological crosscontamination of Mars, the astronauts, the samples, and Earth.

\footnotetext{
${ }^{5}$ At the time of this workshop, work was nearly complete on the development of a Draft Protocol for testing and handling returned martian samples. The Draft Protocol has been reviewed and published since that time, and is considered the conceptual framework for the facilities, containment, preliminary scientific analyses and biohazards testing that will be used upon return to Earth with martian samples (Rummel et al., 2002).
} 


\section{Organization of the Workshop}

The Workshop brought together a multi-talented group of 29 professionals from various NASA locations, academic institutions, industry, and other organizations - plus several students - to explore key planetary protection issues and document their findings. The 2-1/2 day meeting was held at the Pingree Park mountain campus facilities of Colorado State University (see Appendix A). The conference environment was found to be quite conducive to a productive Workshop, and provided a level of seclusion and focus that materially aided the group's deliberations.

The objective of the Workshop was to produce utilization-oriented information on planetary protection issues, policies, and systems operations by encouraging a synergistic interchange among participants from NASA and the broader scientific and engineering communities. The Workshop participants (listed in Appendix C) were chosen to represent a breadth of professional expertise and backgrounds so that the system and mission-wide issues would be addressed.

The Workshop was organized to focus on the human exploration of Mars from a planetary protection standpoint (see agenda in Appendix A), with the primary question: Can human exploration be effectively accomplished without forward or back-contamination? A primary reason for a martian mission to include humans is the expectation that astronauts will be able to better explore, identify, and examine scientifically interesting environments on Mars than are reasonably accessible by even advanced robotic exploration. The Workshop sought to address the operations that might be involved in human exploration missions and to bring an engineering perspective on relevant systems and concepts required for effective exploration of remote, possibly hostile, environments. The Antarctic experience on Earth and the exploration of other extreme environments by humans were used as case examples for comparative purposes, including examination of similarities and differences.

At the Workshop, a list of critical issues were identified as important for supporting effective scientific study of Mars through human exploration. These included:

- The required information to be gathered by precursor robotic exploration

- The nature of forward contamination mitigation efforts and systems

- The inevitable human health and back contamination questions associated with astronauts living and working for several to many months on Mars, even though they will be confined within a local supportive environment (base or mobile rover)

- The return of astronauts, martian samples, and at least parts of the life support systems to Earth

- The need for scientific samples from martian environments to be free at all times from Earth contaminants so that information and conclusions from these samples can be valid and accurate.

The Workshop began Thursday morning, June 21, 2001, with a general overview of its tasks and objectives, followed by brief presentations on relevant background topics. These tutorials provided all participants from a variety of disciplines with a baseline familiarity of the various aspects of planetary protection to be considered. The topics and their presenters included:

- Review of Current Status of Planetary Protection Issues and Planning — John Rummel

- What is Life and Where Might We Find It? - Christopher McKay

- What is Mars Like? - Christopher McKay

- The Arctic and Antarctic Experiences - Diana Wall and Dale Andersen

- Why Send Humans into Space? - Gary Martin

- NASA Plans and Concepts for Mars Exploration - Kent Joosten and John Rummel

- Planetary Surface Activities by Humans - Stephen Hoffman 
- Human Health, Needs, and Abilities in Extended Space Missions - Robert Phillips

- Cave Explorations as a Model for Planetary Protection Protocol Development - Penelope Boston

The Workshop was organized to address the critical issues by examining the following topics within working groups, followed subsequently by plenary presentations and discussion:

1. Protecting Mars and Mars samples from forward contamination, Earth to Mars. Preventing contamination resulting from transfer of materials from Earth to Mar; Mitigation procedures and equipment for both precursor robotic and human missions

2. Protecting human health against risks from the Mars environment. Hazards and consequences to human health from anticipated and unanticipated risks; control of exposure to risks during occupancy of the base habitat and exploration operations

3. Preventing back contamination of Earth from Mars Return. Preventing contamination resulting from transfer of materials from Mars to Earth; sampling and return preparation on Mars; procedures upon mission return to Earth

4. Operations enabling a safe, productive human presence in the exploration of Mars. Base habitat features; support and exploration equipment; operating procedures consistent with forward and back contamination control

Other general topics pervasive throughout all the working group and plenary discussions included:

- Human capabilities in the scientific exploration of Mars. Geology, geophysics, exobiology, human physiology; exploration and operational skills

- Risk identification, control and mitigation. Identification of risks and uncertainties; actions to define, reduce, control, and/or avoid these risks

Following the opening session and background presentations, the Workshop participants were organized into the following three working groups (see Appendix B) corresponding to the first three topics noted above:

WG1 - Protecting Mars and Mars Samples from Forward Contamination "Protecting Mars and Science"

WG2 - Protecting Human Health Against Risks of the Mars Environment "Protecting Human Health"

WG3 - Preventing Back Contamination of Earth from Mars Return "Protecting Earth"

Participants were assigned to one of these three working groups based on their primary professional expertise and interests. Each working group was assigned a chair/rapporteur and a reporter prior to the workshop. The detailed findings from these three working groups are presented later in this workshop report.

After these three initial working groups reconvened for plenary presentations and discussions, the participants were subsequently assigned to one of two parallel working groups dealing with planning logistics and operations (also in Appendix B). Each of these new working groups was comprised of a mix of members from the three initial working groups. The topics of both working groups were the same: 
WG4A - Enabling a Safe, Productive Human Presence in the Exploration of Mars

"Mission Operations I"

WG4B - Enabling a Safe, Productive Human Presence in; the Exploration of Mars

“Mission Operations II"

Each of these two "Operations" working groups was asked to include at least the following operational procedures in their deliberations: (1) distant surface sample collection; (2) sample analysis; (3) in situ resource utilization (ISRU) at the base; (4) plant-growth experiments and greenhouses; and (5) subsurface sampling at both $10 \mathrm{~m}$ and $1 \mathrm{~km}$ depths. Both of these Mission Operations working groups were also asked to devote the last hour of their working group time to exploring the question of "what if we find life during the first human mission to Mars?"

Later, after a plenary session devoted to reports from each Mission Operations working group, there was a follow-up discussion that included a full-group discussion of that question. The concluding plenary session time was devoted to receiving input from all participants on overall Workshop recommendations and conclusions.

The report sections that follow provide overview comments covering the general workshop topics, followed by individual reports by the five working groups, as compiled by their assigned rapporteurs and reporters. The Workshop Report closes with a summary based on the conclusions and recommendations discussed in the final plenary session. 


\section{Overview Comments on the General Workshop Topics (Marvin Criswell)}

The purpose of this section it to address some general and underlying topics which were pervasive in the workshop discussions, but which were not specifically identified in the working group and plenary discussion format of the Workshop. These comments are from the viewpoint of the hosting member of the organizing committee. His background is a good illustration of one general objective of the workshop, namely, to initiate and facilitate discussion and "brain-storming" on the broad workshop topic among interested individuals with varying breadths and depths of expertise in relevant subjects.

The workshop participants included an eclectic mix of backgrounds and expertise. Some participants were world experts in specialized science areas, but had not previously examined in significant depth the overall planning and operations issues of a human exploration mission. Other participants had limited science backgrounds, but were strong in engineering, planning and/or operational issues, including several who were active in the present human space program. The lead editor is a structural engineer who has worked on inflatable habitat structures and other infrastructure for future martian or lunar bases. Several other participants had extensive background in planetary protection and had contributed to the development of the Draft Protocol for handling and testing returned martian samples. This wide diversity of perspectives and expertise among participants greatly facilitated the lively discussions and 'brainstorming' on the broad issues involved in the workshop. The process of blending specialists in many fields with the broader considerations of planetary protection contributed to productive discussions and useful recommendations that are supportive of mission needs and placed in the proper context.

\section{Workshop characteristics and tasks}

A wide range of subjects and considerations relevant to early human missions to Mars and the planetary protection perspective were addressed in the discussions and recommendations of the workshop sessions, working groups, and plenary meetings. At the Workshop, no definitive description of a future human mission was provided or assumed in discussions. The groups considered a generic mission architecture and design, and assumed there would be a balance of science and base development objectives, but they had no specific details on landing site location, time schedule, or many other details. Also relevant but not specifically available were details on technologies which surely will be accessible to those constructing and carrying out a human mission to Mars, including the means and technology for roundtrip transportation of the crew, living quarters, life support systems, surface transportation, operational equipment, supplies, sampling techniques and assessment procedures. Discussions, both within NASA and in other organizations interested in Mars missions, have provided many concepts and ideas on how such a mission might be planned as well as information on some of the challenges that must be met well before the detailed planning and preparation for such a mission can proceed. Even though much of the information is preliminary and exploratory in nature, the planning for a human mission to Mars has progressed to the point where planetary protection issues can be identified and discussed along with the major mission requirements under consideration.

The organization and role of the Workshop reflected its very broad overall charge and the lack of welldefined mission architecture to examine and help plan. Thus, the role of the Workshop was to initiate more dialogue on the overriding issues of "Is there life on Mars? If so, where and how can we find it, what is it like, and how do we respond to it? Is any of this possible life or material on Mars dangerous to the astronauts and to Earth, and if so, in what ways and to what degree? Can a human exploration mission to the surface of Mars help answer these questions and can it be done effectively and safely? Other critical questions included how can human exploration/scientific missions to Mars be conducted so 
that such questions can be efficiently and accurately answered without being hindered by unreasonable mission constraints and while also avoiding unacceptable risks to Mars, the astronauts, and to Earth? The Workshop also considered: What are the areas of largest concern, and how should we be addressing the identification, control and mitigation, and, when possible, the avoidance of these risks?"

A key and intended product of the workshop is information that can be built upon in subsequent efforts, including additional workshops that consider planetary protection issues in human space exploration missions beyond the neighborhood of Earth. A basic goal of this workshop and any subsequent workshops, reports and planning efforts is to foster an awareness and appreciation of planetary protection issues by those involved in formulating mission plans, thus facilitating the inclusion of the planetary protection concerns into mission organization and planning in an effective and timely manner.

\section{Human capabilities, challenges, and considerations in the exploration of Mars}

The exploration of Mars (and other relatively nearby locations within our solar system), including the associated search for non-Earth forms of life, will almost certainly be a combination of robotic and human efforts. Robotic exploration missions form the first phase of this exploration, with their surveillance, remote sensing, photography, mapping and small region surface explorations that have already begun. Future robotic sample return missions will contribute valuable scientific information as well. Although the capabilities which can be provided to robots is continually increasing, the predominant viewpoint is still that a later human exploration phase, conducted in conjunction with accompanying robotic equipment, will bring powers of observation and decision making not anticipated from robots within the expected time frame of a first human mission to Mars. Aside from the human desire to visit and explore the unknown, there will be valid reasons related to exploration efficiency and thoroughness that will almost assuredly require a human presence. Undoubtedly, there will be many complexities as well. Some of the other issues associated with future human-robotic exploration are discussed below.

Communication Time Lag: One important and challenging limitation of physics for robotic exploration of Mars is the delay in the Earth-Mars communication time due to the large distance between Earth and Mars. On Earth, no such delay (beyond a few milliseconds) hampers the interactive control and operation of robots in even hazardous and inaccessible locations. Depending upon the relative position of Earth and Mars in their orbit, the one-way communication time from Earth to/from Mars is many minutes to a few hours. This delayed feedback time makes interactive control from Earth of robots on Mars to be sluggish at best. The degree of autonomy and decision-making skills needed by a robot on Mars to be an effective and thorough explorer are very high. A human explorer on Mars with professional judgment and training to make onsite the relevant exploration and operation decisions does not have this limitation. Until a very high degree of exploration intelligence is autonomously provided within a robotic explorer, the human explorer will be better equipped to deal with the unexpected, both opportunities and dangers. An astronaut explorer on Mars can also very effectively interact with on-site robotic equipment, as no significant communication lag time would exist between the human and robot under these conditions.

Precursor Information for Planning: Although expected requirements for robotically-obtained information needed prior to a human mission were not detailed at this workshop, it was broadly assumed that available information from robotic precursor missions would include at least extensive mapping, photographic and other remote sensing imagery, site surface geological and chemical analysis distributions, and general atmospheric (including dust and wind data) information. High resolution information is expected to be available in the immediate vicinity of the chosen site, with this information probably including the results of at least some shallow drill sampling and possibly surface sample return specifically from the chosen site. Information on the probable amount, form, location and availability of water and other potentially useful local materials would be helpful in mission planning, including in an evaluation of risks and opportunities. Any available information supporting the probable presence or lack 
of martian life, either in general or at the planned site, would be of great interest and utility in assuring the initial base will be located at a relatively benign and biologically inactive site.

Human Contamination Concerns and Isolation: The introduction of humans and their capabilities into the exploration of Mars comes at the price of greatly adding to the complexity of the exploration process, both in the mission scope and operations and in the magnitude of the planetary protection issues. Each human is host to a complex bacterial community related to digestion and many other physiological functions. All components of a human mission to Mars, most notably the astronauts themselves, cannot be sterilized before launch. Rather than a preemptive strategy of outgoing mission sterilization, the strategy must be one of transporting a complex ongoing Earth life system, acknowledging that it will be far from sterile, and isolating it from the Mars environment despite close contact during the mission. This isolation needs to be extended to the astronaut exploring the vicinity around the base, including while taking samples and helping operate drilling or other equipment.

Handling Diverse Contaminants: A successful human mission will involve the return to Earth of astronauts, scientific samples and a complex of life support systems. The visiting life system comprised of humans and their associated life support systems must be isolated from the Mars environment during the mission to avoid harmful contamination both while on the planet and upon return. During the multiple-month travel time to and from Mars, as well as during the stay on Mars, this visiting system of human life and accompanying organisms will be generating biological contaminants through respiratory, food system, and human waste production. Some of these contaminants can be minimized, but not totally eliminated, through recycling. Recycling will be highly advantageous and possibly necessary to limit the mass that must be transported to Mars, although it also involves additional equipment and possible contamination sources.

Long Duration Issues: The long transit and on-site times (relative to the Apollo moon missions) complicate the mission operational tasks that are needed to maintain isolation of the indigenous martian environment and the visiting Earth life systems. Mars missions will be unlike the relatively brief human visits to the Moon that were handled in way analogous to a backpacking trip, with all supplies transported in a finished form, used with minimal recycling, and carried back after a brief visit. Astronauts on the first Mars mission will face the psychological and planning challenges of an extended mission, including basic life support issues of food supply, breathing oxygen, control of human wastes (liquid, solid, carbon dioxide, etc.), issues with great operational challenges and contamination potentials. Although the likelihood of in-flight illness from at least contagious illness can be minimized by pre-flight quarantine, even robust, well prepared astronauts on the first martian expedition will not be immune to medical illness, psychological distress, and accidents, all of which can have both operational and contamination control impacts.

Apollo Experiences: The Apollo missions to the Moon involved the contact of humans with nonterrestrial material and associated concerns about contamination. While the moon was anticipated to be lifeless and hostile to life, procedures for on-site contaminant control, post-mission quarantine of the astronauts, and handling of returned samples were still included as a part of the mission planning prior to the launch. These past Apollo experiences have some relevance to the first human mission to Mars. However, because the planetary conditions on Mars are known to be much more conducive to life than the moon, at least locally, planetary protection is a much greater concern on Mars. Thus, the Workshop discussions referred more often to the planetary protection protocols then being developed for robotic missions rather than to specific Apollo experiences. 


\section{$\underline{\text { Identification, evaluation, and mitigation of risks }}$}

One of the key questions regarding planetary protection is the generic underlying question applicable to many systems, namely, "how safe is safe enough?" Decisions about assessing and controlling risks follow after this basic question has been addressed in at least qualitative terms. The first human mission to Mars will entail a large number of different risks to different parties, with a wide range of appropriate target risk levels. The selection of tolerable and acceptable risk levels depend upon the unavoidable hazards involved, the characteristics of the hazard, and very much upon the consequence of a failure. These same principles are involved in a wide range of activities, from the selection of structural design procedures to aircraft and infrastructure operations, and space missions. They will likewise be important questions on a planetary scale when considering potential risks to Earth and its inhabitants from possible harmful cross contamination.

Another class of risks comprises those faced by the individual astronauts in future human exploration missions. From ancient times through the present, exploration has been an activity where significant risks have been accepted. Exploration usually involves venturing into unpredictable and at least partially unknown environments using relatively complicated transportation and support systems that are often not completely proven and commonly push the state-of-the-art of the sponsoring group or society. Explorers of the New World in the 1500's, the Polar Regions in the early 1900's, and more recently deep-sea explorers, Apollo astronauts and others in space programs worldwide have all operated in environments of risk that would be unacceptable for more routine activities like commercial air travel or driving. Test pilots and mountain climbers are among other "explorers" who must accept relatively high risks as an unavoidable, undesirable, and only partially controllable price associated with pushing back the known frontiers. True explorers, as opposed to risk seekers, act to minimize risks and avoid failures to the extent possible with practical means. They consider the acceptable risks in relation to the mission needs and objectives. These are not easy assessments to make, and are even more difficult when the risk levels and factors are incompletely known, as they will be during human missions to Mars.

The first mission to Mars can hardly avoid a significant level of risk. It will involve a high degree of venturing into the unknown using a complex transportation and life support system with many advanced and state-of-the-art features in what, by definition, will be the first actual use of the complete system.

Regardless of the development effort and the amount of component and prototype testing, the first human mission will be the initial experience of fully operating the complete system for its intended purpose.

Risk factors associated with planetary protection concerns will be among many project risks that must be identified, defined, understood, and evaluated, and then reduced, mitigated, or eliminated as much as possible. Although safety of the overall mission is paramount, detailed analyses of risks also need to be accomplished at the component and sub-system levels. This examination of the smaller scale pieces, along with an examination of their role in the overall system response, are necessary input to assuring that the overall and systems risks are reduced to a level deemed acceptable considering the consequences of failure in each and every possible distress mode. In the initial stages of project definition and planning for human missions, these philosophies of "identify, evaluate, reduce" and "link acceptable risk to possible consequences" are very useful "thought process" tools for qualitative examination of project characteristics, even though the risk assessment at this stage certainly cannot be quantitative. It was with this approach that workshop Overview Comments on the General Workshop Topics. 


\section{Working Group Reports}

This section provides a summary of the findings and recommendations of each of the five working groups, including

1. The general charge to the working group

2. The membership of the working group

3. A summary of the group's actions

4. The report of the group's rapporteur and recorder

Each working group prepared and submitted a detailed report that reflects input from the group during its deliberations, as well as discussions with the entire workshop in plenary sessions, evening meetings of some of the workgroup members, and extensive work by both the rapporteur and recorder in integrating and documenting this information.

The working groups approached their tasks, formulated their discussions, and structured their reports in various ways. Thus, the working group reports below differ in style and length. The topics assigned were too extensive and complex to be described completely in the time available. Because all working groups faced time constraints, the individual reports represent an overview of the topics identified as most important and relevant by those in the group, and do not represent complete and definitive studies of the assigned topics. The findings and reports below are intended to provide important preliminary information and input to subsequent workshop and planning efforts that undoubtedly will occur well in advance of the first human mission to Mars.

\section{Working Group WG1 - Protecting Mars and Science: "Protecting Mars and Mars Samples from Forward Contamination"}

\section{Charge: To identify the risks of most concern in protecting Mars from the unintentional introduction of terrestrial life; to prevent contamination of otherwise pristine Mars samples with contaminants of Earth origin; and to identify general mission characteristics and operations that can address these concerns consistent with mission objectives.}

\section{Working Group Members:}

\author{
Penelope J. Boston, Rapporteur Fred Rainey, Recorder \\ Dale Andersen \\ Frank Grunthaner \\ Danielle Prieur \\ Diana Wall \\ Charles Barnes \\ Donald Henninger \\ Pericles Stabekis \\ Katie Harris, Student
}

\author{
Dean Eppler \\ Mark Lupisella \\ Russell Vreeland \\ Melinda Miller, Student
}




\section{Preliminary Remarks:}

Among the parameters examined and identified by the group, as a key issue relevant to forward contamination concerns is how conducive the conditions are at the specific site of interest for both the support of martian life and any organisms of Earth origin, which might be brought to the site. The group proceeded to define a system for classifying martian sites according to levels of scientific and biological interest, based on a similar classification system of the National Science Foundation for polar region sites of special scientific interest. Characteristics and research needs were examined relative to forward contamination issues for a human mission to Mars circa 2020 and a later mission as well. Although the group addressed forward contamination primarily, the proposed classification and mapping of Mars sites is applicable for many more uses, including planning of mission operations and sampling. This broader applicability is true for other topics addressed by the group and illustrates how the deliberations of all three working groups on Protecting Mars and Science, Protecting Human Health, and Protecting Earth were very interrelated.

\section{Working Group Report}

The report of the Working Group WG1 - "Protecting Mars and Science" is organized as follows:

1. Preamble - Philosophy and Approach

2. Extended Essay on Role of Precursor Missions

3. Recommendations for New Guidelines

4. Evaluation Matrix

a. Classification of sites of special scientific interest

b. Identification of contaminating microbial communities

c. Sample evaluation matrix

d. Zones of contamination control

e. Temporal and sequencing issues

f. Human operations

5. Human Mission Scenarios

a. Mission scenario 1 - circa 2020 Human Mission

b. Mission scenario 2 - circa 2050 Human Mission

6. Research Needs

a. Research questions most pertinent to a near-term mission, circa 2020

b. More general mission research needs

7. Research Venues

a. Ground-based

b. Existing planned precursor missions

c. Transition precursor missions. 


\section{Preamble - Philosophy and Approach}

Philosophy: The Earth is not an inanimate object. It is a living, breathing, active orb. Its life forms have expanded in number and adaptively radiated to now reside throughout the planet. Living things are as much a part of the Earth as is its molten core, its volcanoes, and its rocks. Humanity, as the most technological life form yet evolved on Earth, has always channeled its energies and biological desire for expansion into exploring the unknown on our home planet. The next step in that process may well be the exploration of Mars.

As preparations begin to explore this other world, indeed, a world that may already possess another biosphere, this overall exploration effort is compelled to exercise whatever measures are necessary to protect that putative life from any deleterious effects of our exploration, scientific inquiry, and other human activities that may be conducted on Mars. For the integrity of our scientific investigations, we must prevent contamination of samples taken in various ways and from various places. For the preservation of biodiversity in our Solar System, we must err on the side of caution and conservatism in our planetary protection planning, while still enabling the productive exploration, investigation, and utilization of Mars by our species. This is a significant, but not insurmountable, challenge when approached with care, imagination, and our current and foreseeable levels of technology.

Approach: Planning human missions with care to "protect Mars and science" involves considerations of many parameters, including how the design and preparations for the mission are conducted, what is known from precursor missions, what is brought to Mars, where the landing is to be, and what are the planned activities during the exploration. A key parameter is how conducive the conditions at a specific site may be for both the support of martian life and for any introduced organisms of Earth origin. The Working Group discussions first emphasized the identification and understanding of these spatial areas of high biological interest, followed by considerations of the classes and origins of possible contaminants, their possible behavior and impact in the martian environment, and other important aspects of mission operations.

\section{Extended Essay on Role of Precursor Missions}

For centuries, humankind has pondered the existence of life beyond Earth. However, only recently have we developed the technological means to actively seek out extraterrestrial life. Mars, our closest planetary neighbor, is considered the prime candidate to harbor life in our solar system. The search for life is essentially the search for liquid water, and Mars exhibits evidence of a warmer and wetter past, similar to the conditions on the early Earth in which life evolved.

A human mission to Mars will focus on the search for life, but the issue of precisely where to look first must be resolved. Precursor robotic missions to the Red Planet are essential to identify and classify regions of particular interest in the search for both extinct and extant martian life. Using the information gathered from robotic exploration, a human expedition to Mars will advance our knowledge and understanding of that planet, although the risk of cross-contamination is inherent. Nothing in the mission should either intentionally or unintentionally contaminate or destroy potential evidence for life on Mars. For this reason, identifying regions of scientific interest on Mars is critical before sending humans to explore the planet, and developing protocols to study the planet without profoundly altering its environment is paramount.

One advantage of using precursor robotic missions to identify potentially interesting regions on Mars with regards to the search for life prior to human explorations is the ability to maximize scientific gain while minimizing potential for contamination. While robotic missions are not inexpensive, the value of locating 
and classifying zones on Mars for eventual human investigation is well worth the initial investment of energy, time and money. Knowledge of the regions of interest on Mars will allow the mission to be planned accordingly, taking into account the science that can be done there and developing protocols for accomplishing that science in accordance with planetary protection guidelines. In essence, robotic missions will pave the way for effective, efficient and relatively low impact exploration of the Red Planet by humans.

Robotic precursor missions must themselves be safe, low risk, and scientifically viable for the purpose of detecting life before the introduction of the human element, although life detection likely will not be the primary objective of such missions. Whether these precursor missions find martian life, if it exists, depends upon the form, location, pervasiveness of this life, and chance. Precursor missions are visitors to potentially biologically active and/or supportive regions. They and the equipment aboard them must be sterile upon arrival at Mars.

The precursor missions will need to classify sites according to a predetermined classification system. In doing so, sites need to be categorized primarily according to level of scientific interest. This information, along with potential site topographic data and other characterization, will allow for site selection and preplanning of fieldwork and contamination avoidance protocol. Human missions will thus be contingent on the knowledge gained from the robotic missions.

Without liquid water, there can be no life - at least, no life as we know it. Water is the overriding requirement for defining and supporting all Earth-based living systems. It is true that living things require more than simple pure water, materials such as carbon, hydrogen, nitrogen, oxygen, phosphorous and sulfur. These elements must be available in specific ranges of quantities for all living things. It is also true that many organisms can derive one or more of these materials from gases (i.e., $\mathrm{CO}_{2}$ fixation in autotrophy or $\mathrm{N}_{2}$ fixation by cyanobacteria).

A step between exploratory robotic missions and human missions can be transition missions. These transition missions could be used to send small secure samples of microbes into carefully controlled and isolated martian conditions, possibly employing triggered programmed cell death remotely to help assure contamination control. Such missions could also entail monitoring for health effects, toxicology, mutation rate changes, and unanticipated effects. Another study would be the survival of plants introduced in controlled martian environments brought within the mission lander. This could include planting flowering plants or other indicator plant species and measuring the resulting plant-origin contamination and its viability.

Key questions for the planning of precursor missions in support of later human missions are both the costbenefit relationship of such missions and their assistance in assuring the human mission will more effectively address the planetary protection issues with reduced risks. In general, more and better precursor information will allow a most efficient, productive human mission to follow. How much precursor information is required before a human mission? How much is optimal? What will be needed - both basic information and what following any surprising discoveries by robotic missions? Why will it be needed, for what purposes? When? Where on Mars should these missions be sent?

\section{Recommendations for New Guidelines}

Planetary protection (PP) guidelines must be expanded from robotic to human exploration missions. Current guidelines pertaining to forward contamination are from an earlier era of technique and an earlier era in our knowledge of contaminating organisms (Puleo et al., 1977). Additionally, our abilities to detect and quantify microbial and organic contamination are greatly advanced over the state-or-the-art when 
previous guidelines were created. Improved guidelines are needed which also update numerical targets in light of these new methodologies, and they should also include chemical contamination limits.

This working group advocates a rigorous but flexible set of guidelines that can evolve with the changing knowledge base from precursor missions, early human missions, on through to advanced human missions. Based on these considerations, key recommendations and areas of concern are described in the next sections of this working group report.

\section{Evaluation Matrix}

The WG1 group identified a series of items, each of which bears on the specific measures that must be employed to accomplish planetary protection goals. Using these overlapping sets of classifications and concerns, individual mission scenarios can be assessed and planetary protection needs predicted. The following is a description of these individual topics. An n-dimensional structuring and analysis of these items could yield information on relative degrees of difficulty and sensitivity.

\section{a. Classification of Sites of Special Scientific Interest}

Patterned on the classification system on the National Science Foundation (NSF) Polar Program's descriptive system for SSSI's (Sites of Special Scientific Interest), WG1 proposed that martian sites be described according to the metric of potential biological interest. Not only does this reflect inherent relative value for the purpose of detecting life, it also gives insight into the nature of sites that can be considered to have a particular level of biological sensitivity.

Categorizing the sampling sites on Mars may facilitate development of methodologies necessary to meet sterility requirements by the means most appropriate to a specific site or type of site. A single set of numerically specified requirements is advocated as a goal of contamination control requirements. It should be recognized that the means of achieving these standard levels might differ markedly for different types of sites.

As can be seen from the list below, the ordering of the sites, Class 1 through 5, is based upon increasing probability of long-term existence of liquid water and a progressive similarity to Earth-like conditions. Conversely, the sites have been divided into two broader categories. The first functional group is comprised of those with no or little biological interest because the survival of living materials there is at best improbable (Classes 1 through 3 below). The second group (Classes 4 and 5 below) includes sites of significant and intermediate biological interest because survival of life there is either possible, highly probable, to almost certain.

Importantly, this working group recommends that robotic precursor missions be prepared to identify all classes of sites listed below. Planning for human missions would be affected by data from previous robotic missions, which allow the designation of martian microenvironments into one of the five classes. Robotic in situ science can probably be performed on Class 1, 2 and 3 sites. However, any Class 4 or 5 site investigations should be deferred to future missions. Retooling and retraining for future missions to Class 4 and 5 sites will help prevent mistakes that may be made in early exploration of these biologically sensitive areas.

In this context, robotic precursor missions and human missions containing robotic reconnaissance capabilities are essential elements of exploration. Without such information, adequate protection of the martian ecology will be impossible. 
The field handling techniques described below for each class can be performed by either direct human activity or by robotic devices, or by a combination of humans and robotics.

The proposed system for classifying martian sites according to special scientific or biological interest includes these classes:

\section{Class 1 - No inherent biological interest}

e.g., pulverized, weathered regolith, dust, granitic rock and other igneous features, volcanic materials (excluding lava tubes). digging arm.

Field handling techniques: Low technical level needed - picked up by hand tools, by shovel, by

\section{Class 2 - Potential Biological Interest, including Fossil Preservation}

e.g., sedimentary rock, materials showing evidence of past water, rocks containing different colored inclusions, material of non-obvious density or mass, putative biofabrics or biostructures like stromatolites, potential biominerals (e.g., biohematites, highly oxidized manganese minerals, elemental sulfur deposits).

Field handling techniques: Preserve the sample integrity. Field examination, e.g., with hand lens, microscopy, spectroscopy, or other non-invasive techniques for surfaces only. Protect from damage during containment and transport.

\section{Class 3 - Moderate Biological Interest}

e.g., evaporates $\left(\mathrm{NaCl}, \mathrm{CaSO}_{4}\right.$ crystals, quartz, microcrystalline aggregates, carbonated, desert varnish, unusual exfoliation patterns on rocks.

Field handling techniques: For medium levels ( 10-2), preserve integrity and maintain water pockets. Return to laboratory (on-site module or Earth lab) for high level (>10-6) sampling. Transport as separate samples in clean sealed containers.

\section{Class 4 - High Biological Interest - Materials where present life is possible}

e.g., standing liquid water, transitory artesian flow, permafrost or other ground ices (e.g., clathrates), aquifers, small caves, lava tubes sealed by collapse, organic compounds, keragens.

Field handling techniques: High level of sophistication is needed. Consideration is to be given to the appropriate mix and sequencing of robotic, human, and robotically-aided human collection.

\section{Class 5 - Intense Biological Interest - Live organisms or site where Earth-life could survive today}

e.g., deep caves or fissures containing protected pools or surface moisture, "high" atmospheric pressure (above the triple point), warm water, lichens on rocks, endolithic organisms, liquid water at the bottom of boreholes from active drilling.

Field handling techniques: Avoidance of interference with the site takes precedence over all other concerns. Secondly, extreme care to preserve the sample integrity must be exercised. Highest levels of sophistication possible must be used. Carefully consider the combination and sequencing of robotic and human operations. Depending upon the specific conditions, consider the option of leaving the site alone until further protocol development can occur, protocol which is more suitable to extraction, sampling, and analysis of the items of interest, even should this action defer investigation to a future mission. 
This classification scheme is summarized in Table 1.

Table 1. Classification of Martian Sites According to Level of Special Scientific or Biological Interest

\begin{tabular}{|c|l|l|l|}
\hline $\begin{array}{c}\text { SSSI } \\
\text { Class }\end{array}$ & $\begin{array}{l}\text { Level of } \\
\text { Interest }\end{array}$ & $\begin{array}{l}\text { Physical characterization of } \\
\text { the site }\end{array}$ & $\begin{array}{l}\text { Appropriate field handing } \\
\text { techniques }\end{array}$ \\
\hline 1 & Very low & $\begin{array}{l}\text { Pulverized, weathered regolith; } \\
\text { dust, granitic rock, volcanic } \\
\text { materials (other than lava tubes) }\end{array}$ & $\begin{array}{l}\text { Low technical level acceptable } \text { - hand } \\
\text { tools, shovel, digging arm }\end{array}$ \\
\hline 2 & Low & $\begin{array}{l}\text { Sedimentary rocks or rocks with } \\
\text { colored inclusions, materials } \\
\text { showing evidence of past water, } \\
\text { Putative biofabrics or } \\
\text { biostructures, potential } \\
\text { biominerals }\end{array}$ & $\begin{array}{l}\text { Techniques that preserve sample } \\
\text { integrity. Field examination or other } \\
\text { non-invasive techniques for surface } \\
\text { inspection. Protect sample from } \\
\text { damage during containment and } \\
\text { transport }\end{array}$ \\
\hline 3 & Moderate & $\begin{array}{l}\text { Evaporates, crystals, quartz, } \\
\text { microcrystalline aggregates, } \\
\text { carbonated materials, desert } \\
\text { varnish, rocks with unusual } \\
\text { exfoliation patterns }\end{array}$ & $\begin{array}{l}\text { Preserve integrity and maintain any } \\
\text { water pockets, Transport as separate } \\
\text { samples in clean sealed containers }\end{array}$ \\
\hline 4 & $\begin{array}{l}\text { Standing liquid water, transitory } \\
\text { artesian flow, permafrost or } \\
\text { other ground ices, aquifers, } \\
\text { small caves, lava tubes sealed by } \\
\text { collapse, organic compounds }\end{array}$ & $\begin{array}{l}\text { Heep caves or fissures } \\
\text { containing protected pools or } \\
\text { surface moisture, local pressure } \\
\text { above the triple point, warm } \\
\text { water, lichens on rocks, } \\
\text { endolithic organisms, liquid } \\
\text { water at bottom of drilled holes }\end{array}$ & $\begin{array}{l}\text { High appropriate mix and sequencing } \\
\text { of robotic, human, and robotically- } \\
\text { aided human collection }\end{array}$ \\
\hline 5 & Extreme & $\begin{array}{l}\text { Avoidance of interference with the } \\
\text { site is highest concern. Extreme care } \\
\text { and highest level of sophistication } \\
\text { must be exercised to preserve sample } \\
\text { integrity. Consider leaving site alone } \\
\text { until further protocol is developed }\end{array}$ \\
\hline
\end{tabular}

\section{b. Identification of contaminating microbial communities}

In the preparation of the Viking mission, spacecraft contamination control levels after sterilization procedures were based on growth levels obtainable by culturing of organisms sampled from the spacecraft surfaces using bacterial spores as indicators of cleanliness (Puleo et al., 1977). These control levels in essence addressed all potential contaminant organisms as a single community. For human mission purposes, this working group believes that there are distinctive contamination source communities whose ecological context and physiological properties are sufficiently different to warrant separate assessment. In addition, the notion of culturability is a tricky one and may not be the property of most significance. No more than perhaps $1 \%$ of all terrestrial microorganisms can be successfully grown in a given laboratory environment. The existence of these "ungrowables" has become known only within the past several decades as molecular biology analytical techniques have come on line. The potential microbial communities should be identified and characterized. These should be folded into any monitoring program envisioned for the human base. 
Hence, it may be very desirable to have a more complex assessment of a classification of microbial communities by origin, such as:

- Spacecraft, robots, etc.

- Humans

- Life support systems

- Others?

For each microbial community, the following questions should be explored:

- Where is it?

- Outside or inside?

- How many cells?

- Known chemical composition?

If a microbial community is sent to Mars:

- Would they survive the journey? What percentage is expected to survive?

- Would they survive conditions on Mars? What percentages are expected to survive under what

- Conditions (i.e., in which of the classes defined above would they survive)?

- Are survival probabilities similar for all species, or would some species become dominant?

- How would they be dispersed? What percent might be dispersed through each mechanism?

Is there a possible impact by the microbial community under consideration on:

- Mars sterility?

- Mars living communities?

- Experiments for life detection?

- Other experiments?

- Safety of astronauts?

The answers to these questions could be: Yes definitely, high probability, low probability, or no!

\section{c. Sample evaluation matrix}

The working group attempted to imagine a matrix-approach cross-comparison of the variables described above to yield useful insights into planetary protection impacts. The suggested matrix, shown in Table 2, if analyzed block by block, could summarize the relative impact level on each of the various classes of site types by contamination coming from various human mission contamination communities as defined above. In the example below, combinations for which there is a significant probability of a high adverse impact if a contamination community came in contact with a specific site type are simply indicated by an $\mathrm{X}$. Although further study is required to complete the matrix, Table 2 indicates how the matrix is intended to describe the various interactions that might occur given the known properties of the communities and site types involved. For example, human skin bacteria and coliforms dependent upon an environment provided by a human host would probably have little likelihood of surviving in a Class 1 (no inherent biological interest) Mars site. Conversely, spacecraft-associated organisms derived from soil or airborne microbial populations on Earth and capable of surviving the transit from Earth to Mars could be disastrous if introduced into a Class 4 or 5 Mars site where water, warmth, and higher air pressure could foster survival and growth. 


\section{Table 2. Evaluation Matrix for Planetary Protection Evaluation and Planning}

$[\mathrm{X}=$ Combinations for which there is a significant probability of a high adverse impact if a microbial contamination community came in contact with a specific site type]

\begin{tabular}{|l|c|c|c|c|c|}
\hline $\begin{array}{l}\text { Possible Contaminating } \\
\text { Microbial Community }\end{array}$ & \multicolumn{5}{|l|}{ Class of Sites of Special Scientific Interest, SSSI's } \\
\hline & Class 1 & Class 2 & Class 3 & Class 4 & Class 5 \\
\hline Spacecraft Associated & X & X & X & X & X \\
\hline Life support Associated & & & X & X & X \\
\hline Human Associated & & & & X & X \\
\hline Others? & & & & & \\
\hline & & & & & \\
\hline
\end{tabular}

Folded into this matrix should be the array of research that has assessed the likelihood of contamination problems from each community versus each type of martian site. The specific content of each element in this matrix will depend upon many mission decisions and details, including at least the three topics next noted.

\section{d. Zones of contamination control}

Contamination risks follow from the location and type of human operations. Two modes of contamination zoning were considered. The first is a gradational model in which a number of zones of increasing sensitivity are identified. The procedures required with this approach would accordingly be gradational. The second model is a simpler two-zone arrangement where the base habitat and associated operational areas are designated as a zone of high human impact, or the "High Contamination Zone." All sorties beyond this 'High Contamination Zone' would be declared as operating in "Full Procedure Zones," where requirements for maximum procedural decontamination would result in low human impact. The strict requirements for "Full Procedure Zones" could conceivably be relaxed later if repeated trips to the site prove it to be of no biological interest.

\section{e. Temporal and sequencing issues}

Time-sequencing of contamination monitoring, decontamination, and associated procedures is important, but is mission scenario dependent. Detailed requirements must be developed considering these procedures when they become more defined for the specific mission.

\section{f. Human operations}

Differing aspects of various possible human operations in an exploratory mission have significant impact on planetary protection issues. Critical future decisions regarding the exact mix and responsibilities assigned to human, robotic, and human-guided robotic sorties all have differing implications for contamination and thus for planetary protection.

\section{Human mission scenarios}

The working group considered two separate scenarios, a near-term (initial) mission and a later mission which likely, but not necessarily, would build upon the experience of one to several earlier human missions, to compare the planetary protection issues at differing stages of human exploration development. 


\section{a. Mission scenario 1 - near-term Human Mission, circa 2020}

Lack of direct knowledge and experience will dictate a uniform containment strategy for all science sites because it is doubtful that sufficient information will be available from precursor missions to allow relaxation of containment restrictions prior to the mission.

This scenario assumes that all communications, positioning, and meteorological systems will be in place as infrastructure on the site by at least one supply landing prior to the human phase of the mission. Precursor exploratory missions include those currently planned, including robotic missions with sample return.

In about 2012, detailed design of this circa 2020 human mission is expected to begin. The following information would be available for the landing site and immediate vicinity: mineralogy, surface chemistry, possible presence of surface and near-surface water, radiation environment, geomorphology, high quality images, in-situ microtextural information, surface oxidation, hot spot detection, and notable local conditions and phenomena. During the mission design period, additional yet undefined precursor missions after 2012 will involve site selection and generation of additional site information.

Delivery of basic infrastructure and supplies, along with the positioning, deployment, activation, and start of functioning of some facilities, will be done in advance of human arrival, with planning for these deliveries and activities relying on the information noted above. It expected that the pre-landed cargo would meet the same contamination control levels for the landing site as appropriate for the later human phase of the mission.

The landing site will clearly be at least locally impacted by the landing operations. Thus, it would be best if the landing were far (perhaps at least $100 \mathrm{~km}$ ) away from highly interesting sites (SSSI Classes 4 and 5). It could be possible to land near sites to be explored by subsurface investigations; such proximity would reduce the distances drilling equipment and crew would need to be transported. Similarly, the base camp (located near to the landing site) will need to be quite far from potential sites of highest interest; again, an appropriate preliminary distance may be about $100 \mathrm{~km}$. These requirements dictate that pressurized rovers with a range of at least a $100 \mathrm{~km}$ (at least $200 \mathrm{~km}$ round trip) will be required to access the more interesting sites.

Many questions exist regarding space suits for human activities in the ambient martian conditions, such as: Are the current suit venting systems application to Mars situations or will they require extensive modification? What level of filtration is possible (e.g., can viruses be removed?) and needed? Can an outer protective garment be placed atop the primary suit when the sampling of sites of possible hazards is being done? Given that there will probably not be great improvements in suit design prior to a 2020 mission, applications of the current suits for Mars need to be evaluated, and then modifications deemed necessary then be designed and implemented.

\section{b. Mission scenario 2 - later mission, circa 2050}

A circa 2050 human mission should be operationally easier than the 2020 mission, even if the initial human mission is postponed until this much later time, due to anticipated technological advances and additional precursor mission information.

The envisioned additional precursor missions would likely yield information much better describing both large-scale and small-scale features on Mars and should lead to modifications for contamination control policy, e.g., criteria for biological assessment at various spatial scales, including specific locations, 
regions, and entire planet, along with information needed to define a contamination gradation model rather than the rougher initial dirty/clean model outlined in Section 4d. Some of the restrictions noted for the 2020 mission, such as distances from sites of special interest, might be able to be reduced based on this additional information, although the basic mission characteristics would likely be similar.

The additional time before this later mission would allow the mission to utilize technological advances either resulting from general advancement of widely-available commercial technologies and those specifically developed for human exploration missions. Computers, robotics, remote sensing and analytical laboratory capabilities are among the technologies that will almost certainly be much more advanced. Additional development of space suits, base life support systems, pressurized rovers, and many other components and systems support human exploratory missions would be expected.

\section{Research Needs}

Among the research questions identified by the working group and possible research directions noted are the following:

1. Life support systems: How will wastes be contained? All the life support activities need to be designed considering preparation of the mission for final departure of the astronauts from the Mars base site. What may be left behind? In what type of containers?

2. How clean must items used on the martian surface (e.g., mobility elements) have to be? How can these level-of-cleanliness design requirements best be defined, provided, and maintained throughout the mission?

3. Broader ecological/environmental impacts (e.g., non-biological environment impacts) possible with a human mission must be studied. Time did not permit the working group to explore these needs in depth.

4. What levels of chemical cleanliness or sterility will be required for each component of the mission? What is meant by chemical cleanliness, what methods can be used for obtaining chemical cleanliness, and how do we monitor the level of cleanliness? Sterilization procedures, including heat, gas, ultraviolet, etc., must be evaluated and compared specifically for use in the Mars human mission context.

5. Exploratory precursor missions are needed and planned to determine pristine baselines of Mars. Are "transition" precursor missions needed that will explore how introduced Earth organisms react to the martian environment (using highly controlled and contained conditions in which Mars material is brought into contact with Earth organisms)? How extensive would these missions need to be, and what information is most needed?

6. If two or more microbial communities come into contact at the Mars site, will they become one homogeneous community over time, even though they began as separate communities?

These and other research issues were further categorized as being especially relevant to either a near-term Mars mission or for later missions and human missions in general.

\section{a. Additional research questions most pertinent to a near-term mission, circa 2020}

Research needs include development of rigorous contamination control technology and procedures consistent with, but not limited to, current planetary protection requirements: 
- Spacecraft cleanliness and isolation of other sources of possible/probable contaminants (humans, life support systems, etc.)

- Contamination control of non-biological sources during human missions, such as from mechanical disturbances (rovers, exhaust/venting, etc.), airborne pollution (such as from industrial chemicals), water, heat, lights, etc.

- Some specific research directions noted include:

- Carbon elimination techniques - although much is now in place, more research is likely needed as this need applies to human mission details.

- Evaluation of ethylene dioxide and other gases for sterilization.

- Evaluation of how much biological and chemical residue is expected and acceptable on cleaned surfaces.

- Monitoring and measurement techniques and procedures. For determining contamination in the environment, what remote sensing techniques are available? possible? needed? What tasks and responsibilities do the human astronauts have? How are human health, including physiological and psychological conditions, best monitored and measured?

- Measurement of how many microbes escape from various designs of astronaut pressure suits due to venting, etc. Measurements of the magnitude of microbe escape by enumerating colony forming units and/or molecular detection techniques are needed. Also needed is work on what materials, either in situ or imported, can be used to produce an effective biocide.

\section{b. More general mission research needs}

Research work needed prior to humans going to Mars includes inventorying and evaluating possible contaminating microbial threats or communities that can or cannot be entirely controllable and might be carried by a human mission to Mars:

- What: E. coli, strep, common cold, etc.

- Where: humans, spacecraft, robots, life support, rovers? Inside the base living/operating unit or outside?

- Levels: possibility of occurrence and magnitude (number of cells) likely.

Research required relevant to contamination on Mars, some of which might be conducted by transition precursor missions and Earth-based research using highly controlled sample return material, include:

- Assessing probability of mission components making contact with Mars material, including perhaps the Mars atmosphere,

- Determining viability of various microbial communities and possibilities of mutations,

- Determining probable rates of growth of such communities and rates of mutations,

- Assessing likely dispersion (localized vs. regional vs. global) of various agents, including mechanisms of dispersion (atmospheric transport, direct contact, etc.)

- Evaluating impact of possible microbial and other contaminants on Mars sterility (including possible interaction (including enhancing of activity) among indigenous life and/or terrestrial organisms).

- Procedures needed to maintain the integrity of life-detection experiments.

- Define possible impacts on indigenous biota, a task complicated by possible Mars life not necessarily being the assumed carbon-based, liquid water requiring organisms and remaining research questions on alternatives to a carbon-based organism.

- Explore and develop possible methods for the detection of indigenous biota by unmasking or activating dormant indigenous biota.

- Define the temporal aspect of the topics listed above; how does time affect all of these? 
The group noted that cave contamination research may help answer some of these questions, as some known caves offer good terrestrial analogs.

\section{Research Venues}

The sites at which the research outlined above should be conducted vary with the topics and include the following for work needed prior to the landing of the first human mission on Mars:

a. Ground-based - Many items on the research lists above are amenable to investigation, at least in part, on Earth, within general research facilities, facilities dedicated to Mars human mission issues, or in physical analogues such as deep caves.

b. Existing planned precursor missions - Existing (i.e., already planned and defined) precursor missions will provide extensive basic chemical, mineralogical, water and ice, and other information of great utility. These and not-yet designed precursor missions can be used to categorize or describe site class distinctions and to carry out biological assessments of locales, regions, and overall planet conditions.

c. Transition precursor missions - The working group recommended interim experiments be included on precursor missions tasked to look at the effects of the Mars environment on completely contained Earth organisms, including axenic plants, microbially symbiotized plants, and microorganisms of various types, both as indicators of Mars environmental properties and for fundamental physiological response.

\section{Working Group WG2 - Protecting Human Health: "Protecting Human Health Against Risks of the Mars Environment"}

\section{Charge: To explore the hazards and consequences to human health from anticipated and unanticipated risks presented by the martian environment, along with control of the exposure of humans to these risks during habitat occupancy and exploration operations.}

\section{Working Group Members:}

Robert Phillips, Rapporteur

Gary Martin

Robert Zimmerman
John Charles, Recorder

Brenda Ward

Lela Criswell, Student

\author{
Albert Yen \\ Howard Perko, Student
}

\section{Preliminary Remarks:}

The assigned topic of protecting human health against risks of the Mars environment" is a topic hard to isolate from general health issues at a remote environment very different from that on Earth. The general need for a local pressurized environment (habitat, space suit, pressurized rover, etc.) for human life to survive on the Mars surface and the general remoteness of the site inherently result in many human physical and psychological health and performance issues that are quite different than on Earth. The determination of which human health issues are due to fundamental differences and what ones are related to contamination and other planetary protection issues is not easy, as the latter may be interrelated with 
the former, and in many ways may actually be a subset of the first. For example, contaminant control is integral with the design of many life support systems, sampling strategies, and exploration forays away from the habitat. Declines in astronaut performance due to any ill health, including psychological problems, increases the possibilities that procedures may not be as efficiently and thoroughly followed, with contamination and compromise of standards more likely.

Because many possible health and human performance issues relate directly to some part of the Mars environment coming into contact with the human occupants, the group explored selected base operations, sampling, and laboratory scenarios that could bring such material in contact with the astronaut, and also contributing to the potential for back contamination. This group addressed in more detail the issues related to support of humans at the martian base, including in the living habitat, than did the other two initial groups. The human health issues associated with martian dust were given special attention since it may be difficult to avoid the introduction of this material into the habitat and little is known about the potential responses of humans to fine, possibly chemically reactive martian dusts. Given the close relationship among the base operations which might lead to contaminants reaching the human crew and those which might also result in contaminants reaching the martian environment, the group also devoted significant attention to topics also related to forward contamination issues associated with the base/habitat and its operations.

\section{Working Group Report}

Early in the first working meeting, it was decided to ignore factors not specific to planetary protection, i.e., microgravity, low atmospheric pressure and atmospheric gas composition. Factors to be considered included ionizing radiation, dust and electrical discharge, dust composition, dust toxicity, and questions related to possible martian life forms. Discussions followed to list topics of concern, with organization of these topics to follow later. The points raised included:

1. A possible hazard with martian dust is that of immune suppression. Dust may result in a decreased human resistance to radiation damage, especially with deeper lung penetration of particles (dust with particle diameters $=1$ to 3 microns). During mission operations, it likely will be impossible to keep dust out of everything. Even if humans going to the Mars surface stay inside a "submersible" habitat and never venture out, they likely will come in contact with some martian materials as dust is brought inside the habitat by sampling and other operations, including on space suits. What are the necessary limits on dust exposure? When martian dust and water is mixed, what is the result? What microenvironment is created by the presence of humans on Mars? Superoxides brought into the laboratory environment plus water may produce reactive products that are very damaging.

2. Operations that involve digging beneath the surface of Mars may result in biohazards. Will there be life forms similar to bacteria and/or viruses there? We are looking for biological evidence of previous life, including at locations below the surface, so we need to proceed as if we expect to find these evidence of life.

3. Many health concerns are not directly related to Mars biohazard and toxicity issues. For example, the microbes that accompany the explorers to Mars may cause sickness, and human dependencies on trace elements may result in problems unless dietary planning pays specific attention to these human needs. 
4. Key health-related questions include: How much can humans be exposed to the Mars environment even independent of possible present or past martian life issues - the surface conditions, dust, water from martian sources, localized special mineral conditions - and with what possible consequences? How much will humans be exposed to martian dust and what practices will be both practical and effective in reducing this exposure?

5. In the base operation, is it possible to seal or otherwise isolate the human occupancy area from other areas, such as a less secure laboratory area, and be assured that that the crew would never need to enter such laboratory areas to repair or maintain equipment or facilities? How complete can such an isolation system be and need to be? Will robotics technology be advanced to where it will be dependably capable of performing needed repair, maintenance and operation functions?

6. The need to fully characterize the site where humans might go is widely recognized. How complete is "fully characterize," and can it all be done remotely? At some stage, geologists are needed to identify, prioritize, put findings into context, and decide where to drill when subsurface exploration is planned. How directly do they need to explore the sites of interest? If the exploration and documentation work can be done slowly, robots will likely be able to do most of all of this work, but can they provide all the site-specific information needed for formulation of informed geological decisions?

7. If a human habitat and its occupants locally contaminate the planetary surface, how rapidly will the contamination spread? For what contaminants will there be appreciable thermal or radiation deterioration and at what time rate? What neutralization of contaminants will occur from desiccation or ultraviolet light? How much of what contaminants (such as aerosols, water vapor and tiny air-borne particles and microbes) might be released and then dispersed by the ambient winds? Similarly, what types and sizes of fine particle contaminants, especially those which might host microbes, would settle to the martian surface during ambient winds but would be dispersed by martian dust storms? The dusty surface of Mars is likely to be chemically reactive and very free from organics. When water, including as a contaminant, is added, oxygen may be released, and when organics come in contact with the reactive dust, they may be broken down. Are any of these reactions important from a human safety perspective?

8. Life support systems, including their design to operate without the production of contaminating byproducts, will require much more attention than they are receiving at this early stage of planning. Closed-loop systems are preferred for planetary protection, and the technology for the design and effective, efficient operation of such a system remains a challenging task. Will the venting of habitat "waste" products, including gaseous materials, result in surface deposits on such base features as exposed optics components, in addition to raising contamination issues? How best can a habitat be placed and positioned on the surface, outfitted, and prepared to be easy to be made operational once it human occupants arrive? How can is be assured that martian dust or other possible contaminants are not introduced into the habitat before its occupancy? Upon the departure of the human crew, may wastes be left behind in the sealed habitat or in sealed containers within or adjacent to the habitat or other base facility? Are the astronaut suits for exploration away from the habitat and operations within less secure areas to be completely sealed suits? What about the rovers and the habitat overall? The synergies and interactions among the different habitat components - people, rover, sampling, habitat, life support systems, etc.- need careful examination.

9. The possibility of any Mars sample or sampling equipment contaminating the human explorer must be addressed and mitigated. If humans are going to Mars to look for life, with plans to drill in the places most likely to support martian life, we must develop technology to identify and deal 
with the possible biohazard issues - including expansion of present life and reviving of relic life to assure that, at a high level of confidence, we do not bring back something that on Earth can be infectious.

A logic tree for handling samples which conceivably might be a biohazard could be as follows: Provide technology for handling and testing while maintaining a BSL-4 biocontainment level, ${ }^{6}$ then verify and test the facilities and procedures. In the planning process, formulate plans that assume extraterrestrial life will be found, which may or may not be hazardous. Expect that the samples from deep drilling will not contact a sterilizing environment before they enter an investigative laboratory. Expect that if you do find life it may be hazardous, and plan the laboratory procedures conservatively. If any evidence is found that a sample might be hazardous, especially if it is something that could possibly infect Earth, be prepared to terminate the analysis unless a reliable laboratory procedure is available to neutralize the hazard in a secure manner.

Later, the Working Group began to organize the primary topics discussed into a matrix including the following risk factors: (a) physical status of the Mars-mission astronauts, (b) human behavior and performance, (c) physical interaction of the astronauts and the base/habitat with the Mars environment including dust, chemical, mechanical, electrical discharge and radiation cosmic, n-backscatter, and other indigenous matter, (d) biohazards, (e) clinical - injury and disease, and (f) protection of Mars from the possible contaminating effects of human activity on Mars - physical wastes, human wastes, life support system effluents, release of Earth microbes, etc. The use of such matrices including risk parameters, concerns, consequences, and mitigation actions is recommended as a way to organize and examine information regarding contamination which is needed in mission planning.

\section{Working Group WG3: Protecting Earth: "Preventing Back Contamination of Earth from Mars Return"}

\section{Charge: To address sampling and sample return preparation consistent with planetary protection requirements and procedures for samples, astronauts, and other mission components upon return to Earth.}

\section{Working Group Members:}

\author{
Margaret Race - Rapporteur \\ John Batista \\ Mark Kliss \\ Jeffrey Carlson, Student
}

\author{
Judy Allton - Recorder \\ Steven Hoffman \\ Brent Sherwood
}

Kent Joosten
Todd Stevens

\section{Preliminary Remarks:}

The working group on "Preventing Back Contamination of Earth from Mars Return" emphasized prevention and control of contaminants coming in contact with the astronauts and other Earth-related equipment and environments during site investigation forays, sampling, sampling handling and sample assessment on Mars. This group emphasized the importance of minimizing or eliminating contaminants at the potential source, i.e., to minimize or eliminate exposure, rather than to assess and mitigate

\footnotetext{
${ }^{6}$ BSL-4 is the most restrictive containment level as defined by the biomedical community and used on Earth for containment of highly contagious pathogens in high security facilities (CDC-NIH, 1999)
} 
exposure. Consistent with this approach is the philosophy of "assume life exists unless proven otherwise" when collecting, handling, and testing samples. Several other sources and paths of contaminants reaching Earth were not addressed in detail, in large part due to time limitations. These included contaminants arising from Mars base operation, contaminants accompanying the crew on the way back to Earth and the specific protocols for the handling of returned samples.

Because precautions needed in sampling and sample handling can differ greatly for non-biological samples compared with those taken from regions that might harbor or support life, the group addressed the need to characterize sites according to their level of potential biological concern. The impact of the discovery of life on various procedures and the importance of more information about potential global scale martian dusts were also noted. The group proposed a system of zoning for permissible human mission operations that combines information on the extent to which a site or zone has been characterized and its inherent level of biological potential and scientific interest. Their suggestion of zoning for permissible operations is very compatible with the area classification system proposed by the first working group. This similarity of thought on area characterization also reflects the close interaction and similarity among procedures to limit or preclude contaminant transfer either to or from the martian environment.

\section{Working Group Report}

The scope of the working group task was defined to include:

1. Sampling on Mars - robotic and human-aided,

2. Sample return and assessment as related to back contamination, and

3. Crew return, quarantine, and evaluation.

\section{Background}

Since it may be impossible to know with certainty whether or not life exists on Mars in the near term, it is important to take a conservative approach to planetary protection controls for human missions. A conservative approach has already been adopted for robotic sample return missions based on recommendations by the National Research Council Space Studies Board that all materials returned to Earth from Mars must be contained and considered as possible biohazards unless and until proven otherwise (NRC, 1997).

After considerable discussion about various human mission scenarios, the workgroup decided that unless and until it is certain there is no life on Mars, all missions with human crews must maintain this same conservative approach. This translates to containment for samples, spacecraft parts and equipment that have been exposed to the martian surface, as well as containment or isolation for returning astronauts. Implementing this approach to protecting the Earth from harmful cross contamination during future human missions will require careful attention to activities and equipment in transit, on the planet, and back on Earth.

\section{Conceptual Approach to Protecting the Earth - Containment and Contamination Avoidance}

Planetary protection requirements are designed to address concerns about possible forward and back contamination, and these cannot be considered to be entirely independent. Working Group 1 of this workshop is addressing forward contamination concerns during human missions. The WG3 group noted 
that forward contamination generally can be avoided by cleaning and removal of contaminants or by the use of suitable barriers to isolate equipment or materials from contact with Mars. It may be impossible to entirely eliminate human-associated forward contamination during human missions. However, it will be assumed that the level of forward contamination caused by normal human metabolism and presence can be controlled enough that it presumably would not constitute harmful cross contamination to the planet per se, but rather would be a matter of scientific concern during the sampling and collection of martian materials.

For human missions, this group advocated that avoidance of back contamination of the Earth be accomplished by focusing on both returned sample materials and crew members separately and in combination.

Back Contamination and Returned Samples: In general, back contamination of Earth can be avoided by containing returned samples or materials exposed to the martian environment, or by sterilizing materials prior to release from containment. For human missions in particular, prevention of back contamination is also based on the ability to isolate samples, some of which might contain replicating biohazards, from the crew and their habitat.

Isolation of samples must begin as samples are being collected, and isolation should be maintained through transport, analysis and transit to containment facilities on Earth. Draft protocols currently under development (Race, et al., 2002) have already addressed appropriate methods for handling and analyzing martian materials beginning on Mars and upon return to Earth. The working group felt that it would be important to adopt these same protocols (or their future refinements) for handling martian samples during human missions. Appropriate biosafety and PPL (Planetary Protection Level) containment of collected samples should be maintained in transit as well as during any sample screening or preliminary science analyses in situ, in field labs, or in the laboratory at the base camp. Moreover, the base camp laboratory should be completely separate from the crew habitation quarters, and methods should be devised to introduce the contained samples into the laboratory through entry ports dedicated for samples only.

Back Contamination and Crew Members: For both robotic and human missions, the operative concern about back contamination is that of exposure per se. For human missions, if methods can be devised to prevent exposure of the crew to uncontained martian materials or environments, then concerns about human-associated back contamination can be reduced substantially, if not eliminated. Using this rationale, if humans are kept successfully isolated from biohazards, they acquire no exposure to biohazards, thus reducing the need for any isolation beyond that required for medical observation upon return to Earth. It is recommended that crew be isolated from the martian environment at all times to eliminate the major concerns about exposure (rather than devising elaborate protocols for assessing the crew for biohazards upon return). It will be important to maintain human isolation from martian materials during scientific exploration as well as routine outside activities at the base camp (infrastructure maintenance, greenhouse activities, repair of equipment, etc.). Martian materials used for agricultural purposes, especially for growing food for human consumption, must be sterilized or otherwise treated to maintain isolation from potential martian biohazards.

This 'non-exposure' approach is consistent with that routinely used in the biomedical community when dealing with known virulent and pathogenic organisms or biohazardous materials.

Under nominal conditions, a combination of primary and secondary containment, combined with appropriate equipment and protocols, are sufficient to eliminate exposure and thereby protect workers and the environment from contamination concerns (CDC-NIH, 1999). This approach also utilizes an extensive baseline of health and medical information in combination with routine monitoring of workers during and after work with known or potential biohazardous materials. Combining 'non-exposure' and 
routine monitoring could be sufficient to address back contamination concerns related to crew members returning from Mars. Biohazard monitoring (details to be determined) could be included in conjunction with the extensive medical testing that will be a part of any human mission. The lengthy return flight virtually imposes a quarantine of suitable duration to detect obvious health impacts that might pose back contamination concerns.

In anticipation of the possibility of a breach in containment, lapse in protocols or presumed inadvertent exposure, the mission architecture must be able to accommodate containment of the crew, spacecraft and equipment until they arrive at a containment facility of Earth. Depending on mission architecture, isolation of crew and vehicle during transfer in Low Earth Orbit may be required. Upon return to Earth, continued monitoring can occur during the anticipated post-flight medical isolation to ensure that no indications of exposure or biohazards are detected. Decisions about release of crew or continuation of medical isolation would be made upon review of medical information on Earth.

\section{Concept of Zoning for Human Mission Operations}

During human missions, collection of martian materials from a variety of locations will be undertaken. Some sites will be of great biological interest, constituting concern about potential biological contamination; other sites or zones are likely to contain materials of no biological interest or associated contamination concerns. To address these differences, the workgroup developed a fundamental concept based on the iterative, incremental definition of operations zones on Mars designated by two considerations: (1) the extent to which a site or zone is scientifically characterized and (2) its level of biological potential or interest. Under this scheme, the following areas or zones have been designated:

\section{Inside a habitat:}

Zone 1. Habitable zone: Areas within structures, vehicles, suits, etc. capable of supporting human life.

\section{Outside:}

Zone 2. Characterized sites or zones - previously studied and characterized in some way (e.g., by precursor missions, sample return, orbital or remote analysis, etc.) sufficient to make a determination of whether the site is

Zone 2a - 'safe' from microbial biohazards and 'cleared' for extensive human operations and activities, or

Zone $\mathbf{2 b}$ - a site or zone with some level or biological potential that warrants limitations on human access and operations.

Zone 3. Uncharacterized sites - zones or areas with insufficient data to make a determination about their level of biological potential or interest. Operations at these sites warrant limitations on human access or operations until they can be characterized more fully.

The designation of these four categories $(1$ - habitable; $2 a$ - characterized/safe; $2 b$ - characterized/limited access; or 3 - uncharacterized) may also be used in combination with the classification scheme devised by Workgroup 1 for characterizing martian sites with respect to forward contamination concerns and level of scientific/biological interest. Figure 1 illustrates a conceptual approach to how mission exploration travels might operate within a characterized region containing Zones 1, 2a, $2 \mathrm{~b}$ and 3 sites. 


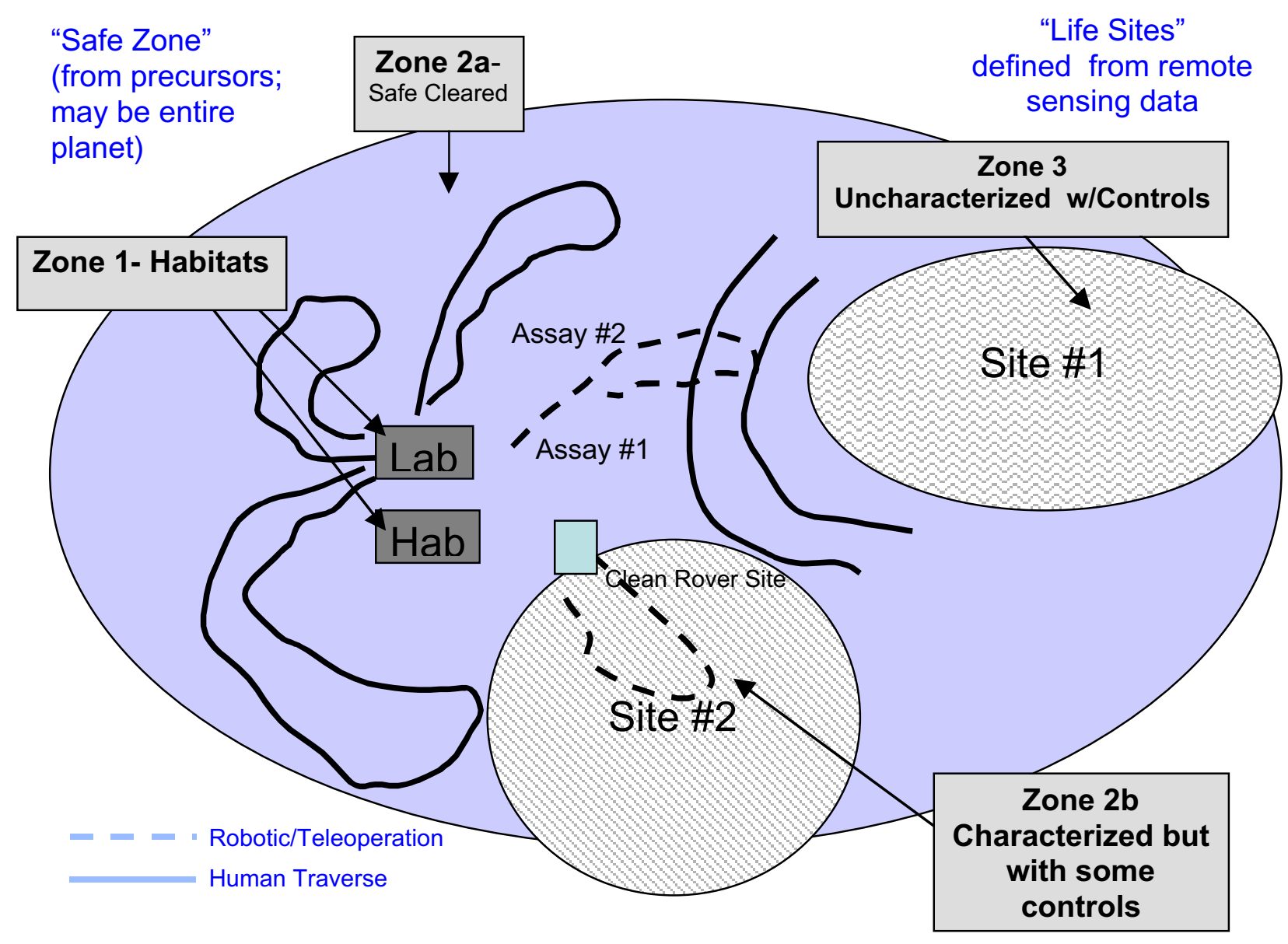

Figure 1. Example of a possible conceptual approach for a site classification system

\section{Designation of 'Safe' Operational Zones}

Using the proposed categorization, zones that are well characterized and are determined to have no indication of biological potential are designated as "safe zones" which are cleared for human activity because they are devoid of martian life. In designated safe zones (Zone 2a), both sampling and routine activities may be conducted without robotic or remote intervention. However, even in designated safe areas, it will be important to maintain the isolation between crew and martian materials. Presumably, the immediate area of the landing site and base camp would be located in a previously characterized area that is of no biological interest and thereby 'cleared' for human activity. This determination will be dependent upon an iterative process using data from precursor missions, returned samples from MSR (Mars sample return) missions, and materials transport models that together help to

1. Define what Mars surface material can be considered globally identical

2. Determine whether this surface material could comprise the first safe zone.

The working group noted that analysis of data from precursor missions and returned samples will be essential to show that dusts and typical surface materials (away from notable features and conditions) down to some depth below the surface are safe from microbial biohazard and thus such surfaces maybe 
cleared initially as suitable zones for landing sites and establishment of base camp infrastructure. In addition, since dispersal of dusts will be inevitable during martian missions, it will be important to verify that these materials do not constitute a biohazard for operations purposes. This safe-zone categorization process would enable planetary protection tolerance of small, manageable amounts of dust inside habitable facilities. Tolerating dust in this manner in turn permits practical operations on long duration human surface missions.

This operation approach concerning dusts leads to two primary operational requirements:

1. Ingress/egress systems that limit the total amount of dust getting inside the habitable zone, and procedures that control and clean away the dust that does get inside. These technologies, then, are not driven by the replicating biohazard back contamination risk, but rather by other engineering and toxicity requirements, including human health. This presumes that future missions and data continue to indicate the apparent 'sterility' of martian surface materials with respect to life.

2. A process of managing the boundary between the safe/cleared zone and other zones that may still pose a back-contamination hazard or remain uncharacterized by incremental investigation. The long-term objective is to shift the boundary so that the safe/cleared zone expands, perhaps ultimately to comprise all of Mars.

The categorization process should be used incrementally to declare ever-widening zones or areas as biosafe for humans. Crews will be sent only to sites deemed bio-safe by previous analyses and characterization. In uncharacterized areas or locations near or within areas determined to present potential biological concern, initial sampling or reconnaissance should be done robotically or remotely in order to determine whether and how future human activities may be safely permitted in the area.

The bounding parameters of sites or zones with biological potential may be designated in a variety of ways - either as a location per se (e.g., a brine seep) or by a combination of important geometrical (latitude, longitude, depth), geological (material composition and mechanical properties) and/or environmental (hydration, temperature) features. The boundary so defined may vary diurnally, seasonally, or with additional information acquired as the operations proceed.

Exploration and sample retrieval within the bounds of zones with biological potential must be done in a manner that maintains the strict separation of the habitable zones from the Mars material. This means that at no time may the materials from a non-cleared site be handled in such a way that they contaminate surfaces that will be introduced into the habitable zone.

This approach drives two principal derived operational requirements:

1. Robotic adjunct exploration and sample collection and handling capabilities which are able to operate within zones of biological potential or uncharacterized sites. Note that the boundary of these restricted sites may be temporarily expanded due to these operations, including extension of a transportation corridor to an onsite laboratory.

2. In situ, field lab or base lab assay capability to determine whether the material represented by the retrieved samples may be redefined to be within the safe/cleared zone (i.e., whether the boundary may be shifted). Ultimately, the goal would be to achieve robotic assay capability at the site of sample collections, so as to minimize necessary expansion of the restricted zone or boundary. DOE/DOD technologies may be directly applicable to meeting this requirement. 


\section{Overall Constraints}

To reiterate, the constraints required to enforce isolation of potential martian biohazards and thereby protect Earth from potentially harmful cross contamination include:

1. Acquisition of samples must be accomplished in a manner that isolates these samples from crew and habitat.

2. All sample handling, screening, analysis and transport must be done under containment.

3. Returned samples collected during human missions will be handled and taken to an Earth Planetary Protection Level-alpha (PPL- $\alpha$ ) facility ${ }^{7}$, just as robotic samples are to be handled upon their return.

4. The crew will undergo follow-up medical isolation coincident with astronaut health analyses and requirements.

\section{Additional contingency constraints imposed due to inadvertent exposure (breach of isolation) are:}

5. Containment of astronauts, spacecraft and samples at appropriate biosafety of PPL levels is required from re-entry through arrival at appropriate terrestrial facilities.

6. Exterior of re-entry vehicle must break the chain of contact with the martian biosphere.

7. Clearance of crew to be determined based on analysis of samples and medical testing.

\section{Needed Technology and Science Research and Development}

Technologies that need to be developed to prevent back-contamination of Earth by biologically active agents include:

1. A suite of technologies must be developed that will facilitate analysis of uncharacterized areas of Mars which hold the potential for supporting life. These analyses will be conducted on Mars either in situ, in the base camp laboratory, or in a mobile facility. This technology should be comprehensive enough to provide reasonable assurance whether samples contain extant life. It is expected that analysis of Mars samples returned from missions conducted prior to a mission with humans will guide the development of this technology.

2. The technology must be in place to limit exposure of the crew and their habitat to the martian environment. Strict separation of humans from potential hazards minimizes planetary protection concerns as they relate to possible back contamination of Earth by the crew and the return vehicle. There is a need to:

a. Make improvements in systems for robotic sampling at sites with the potential for life that integrate human control and decision making into the design,

\footnotetext{
${ }^{7}$ PPL designations (described in detail in the Draft Protocol (Rummel et al.,2002)) are specially defined laboratory containment requirements that combine strict biocontainment (as defined by NIH-CDC, 1999) with highly clean laboratory conditions suitable for protecting sample materials from terrestrial contaminants during handling and testing.
} 
b. Design or modify tools to be used by humans that will allow sample retrieval and minimize the risk of exposure,

c. Design or modify sample transport containers, transfer ports, examination boxes, and laboratory space (with air cleaning/filtering) that prevent exposure of the crew and habitat to sample materials, but allow necessary scientific study of the sample, and

d. Develop or refine suit technology that limits contamination of the crew habitat (including perhaps exo-suits and rovers and suit locks), but can be easily cleaned and maintained.

3. Precursor missions need to establish, as a high priority task, whether the martian dust is global in nature and is or is not a biohazard.

4. Additional Research Areas - All efforts to maintain a barrier between the martian environment and the crew depend on the crew's compliance in strictly following a separation protocol once that protocol has been established. There is, therefore, the possibility that the crew will intentionally violate the protocol, creating a problem that impacts planetary protection. It is suggested that studies of the psychological stress of long-term missions on crew performance include an evaluation of this potential problem and its possible solutions.

\section{Workshop Group WG4A and WG4B - Operations I and Operations II: "Enabling a Safe, Productive Human Presence in the Exploration of Mars"}

After the reports and discussion of the first three working group reports early the afternoon of the second day of the Workshop, the participants were reassigned to one of two parallel working groups each charged with examining the same list of several operational tasks of an initial human mission to Mars. Each of these working groups on operations were assigned a mix of members from each of the three initial groups.

Charge: To explore several operation issues including habitat design, support and exploration equipment, and base operating procedures consistent with forward and back contamination control. A more specific assignment made at the Workshop was for the two "Operations" groups to include consideration of the following six scenarios for issues of back and forward contamination:

\section{Distant surface sample collection}

\section{Sample analysis}

3. ISRU (In situ resource utilization) at the base

\section{Plant growth experiments and greenhouses}

\section{Subsurface sampling (both $10 \mathrm{~m}$ and $1 \mathrm{~km}$ depth)}

\section{What do you do if and when you find life?}

Both Working Groups were instructed to devote at least the last portion of their meeting time to a discussion of the sixth issue: What do you do when you find life? In their reports on Topic 6, the two groups mixed in some comments on the identification and detection of martian life, such as: "what are we looking for and how will we know if we have found it?" How is life to be defined and what forms 
might it take, given that it almost certainly would be of small scale and may be very different than Earthbased life? If such unknown life, either extant or extinct, exists and is encountered, how can it be reliably detected and characterized?

Discussions within the two working groups developed along somewhat different lines addressing somewhat different topics, and thus the two reports are complementary, not duplicates. These differences also can be interpreted as evidence that the two groups had time only to begin to explore the complexity and issues central to the planning of the first human mission to Mars.

\section{Working Group Members - Operations I}

Mark Kliss - Rapporteur
Dale Andersen
Richard Fullerton
Gary Martin
Margaret Race
Jeffrey Carlson - student

Mark Kliss - Rapporteur

Richard Fullerton

Gary Martin

Jeffrey Carlson - student

\author{
Mark Lupisella - Reporter \\ John Batista \\ Donald Henninger \\ Robert Phillips \\ Perry Stabekis \\ Katie Harris - student
}

\author{
Penelope Boston \\ Stephen Hoffman \\ Daniel Prieur \\ Albert Yen
}

\section{Preliminary Comments}

The two parallel working groups on "Enabling a Safe Productive Human Presence in the Exploration of Mars" chose somewhat different approaches and different emphasizes during their limited discussion time, although each addressed all of the six topics. This first working group identified and listed major considerations and concerns for each of the six scenarios, with more emphasis given to distant sampling collection and to plant growth experiments and greenhouses than to the others.

\section{Working Group Report - Operations I (WG4A)}

The working group's report follows the assignment to examine the following six scenarios for issues of back and forward contamination:

1. Distant surface sample collection

2. Sample analysis

3. ISRU at the base

4. Plant growth experiments and greenhouses

5. Subsurface sampling (10m and $1 \mathrm{~km})$

6. What do you do when you find life?

The process used was to identify the core thoughts, indicate why they are important, and then try to suggest examples where possible. Major points of the resulting wide-ranging discussions are as follows: 


\section{$\underline{\text { Issue 1. Distant Surface Sample Collection }}$}

\section{Everything should be "cleanable" in-situ to meet TBD (to be determined) specifications}

The issue of exactly what these specifications for assuring cleanliness will be is obviously critical and must be addressed by further consideration and research. However, the group's overall judgment, consistent with Working Group 1 ("Protecting Mars"), was that if a human mission occurs prior to the relevant additional knowledge being obtained (e.g., perhaps within the next two decades), then the present planetary protection guidelines for forward contamination should apply with added requirements for all associated human-related disturbances - some of which are mentioned in this report and other working group reports from this workshop.

\section{Employ remote assessment of the site in question}

Remote assessment of the site in question, particularly its biological status, should be conducted before sending humans directly to that site. Advanced robotic reconnaissance via telerobotic, semi-autonomous, and autonomous scouts will enable this assessment.

\section{Conduct research on suit contamination containment technologies and procedures}

Research on suit contamination containment technologies and procedures will be important once contamination containment requirements are better understood. Outgassing of both biological and nonbiological sources will have to be addressed. Suits will need to be extremely well cleaned to TBD specifications before and after life-detection activities, but perhaps less so for routine operations. An example of a research question is the following: How will decontamination processes work on Mars? "Real-time" contamination monitoring devices (e.g., carbon or oxygen monitoring devices) can directly inform about the extent of any contamination exposure (both for the environment and human/habitat health) and directly communicate decontamination needs for any given situation.

\section{Route identification and establishment}

Route identification and the establishment of safe, reusable routes to and from key sites could be important for minimizing mechanical disturbance effects at those sites. For example, the directions of approach to the site could be important and would have to be maintained in a way that best preserves the integrity of that site.

\section{Cleanliness and procedural requirements may vary with sampling location and techniques}

Cleanliness and procedural requirements may vary as investigation and collection approaches vary. As an example, a robot, a pressurized rover, and a human explorer may each present unique challenges that could result in at least procedural differences, if not differences in acceptable levels of contamination. For example, the microbial contamination associated with humans may be found to present different threats to indigenous biota compared to the microflora of a cleaned robotic vehicle. Research in the area of microbial inventory and assessment of possible adverse effects is a critical research issue noted by Working Group 1. 


\section{Issue 2. Sample Analysis}

\section{Everything should be cleanable in-situ to TBD specifications}

As noted above, everything associated with sample analysis should be cleanable in-situ to to-bedetermined specifications. This will affect mission planning, design, and operations because all will have to enable activities associated with achieving high levels of cleanliness.

\section{Assume life is present in the sample}

An overall guideline should be to assume life is present in the sample until proven otherwise. All protocols should be based on this guideline.

\section{Sample isolation}

Samples should be kept as isolated as possible at first, and as appropriate as more is learned about the samples.

\section{Non-invasive sample handling}

The sample analysis process should start with non-invasive sample preparation. If at all possible, noninvasive analysis, such as spectroscopic methods, should be done prior to non-consumptive (e.g., nondestructive) methods. Consumptive methods should be used last, after enough has been learned to ensure the integrity of the sample and any life that might be present.

\section{Non-contamination distinctions for different methods}

Sample analysis could be conducted in different ways at the site in question, which might be, for example, an on-site lab or perhaps a remote laboratory. In all cases, the contamination control requirements and procedures for analyzing the sample should be consistent for all labs and for all methods.

\section{Plan for the worst-case}

Clearly, contingencies should allow for the mitigation of worst-case scenarios such as inadvertently introducing radioisotopes into the environment. Certain worst-case scenarios could be "catastrophic" and they should be addressed in the planning process with that in mind.

\section{Issue 3. ISRU at Base}

Contamination sources from in-situ resource utilization at the base were roughly categorized as gases and solids. Two observations for each class of possible contamination source were noted:

\section{Catalog sources of outgassing}

The constituents and amounts of outgassing should be cataloged and assessed for possible impact on the martian environment. Although such outgassing will not likely pose a planetary protection threat because of the small amount of gases involved and atmospheric dissipation and degradation, these sources of outgassing should at least be understood and cataloged, and preferably then assessed for possible impact to indigenous biota, even if that impact is thought unlikely to be significant. 


\section{Use and performance of filters}

If there is a sufficient threat of biological and non-biological contamination from outgassing associated with ISRU, appropriate filtering techniques should be developed and used to mitigate this threat.

\section{Handling of regolith and soil}

Mechanical disturbances should be kept to a minimum consistent with the ISRU. However, large amounts of regolith/soil may be needed, for example, in greenhouses. If martian soil is to be used, it obviously should be done with great caution. For example, if modifications to the soil are required, they should be done in small incremental steps to assess the effect of each step. The amount of processing on the soil should be minimized in order to avoid unknown biological implications.

\section{Extraction and use of ground ice}

A non-invasive biological assessment of ground ice should be done prior to its use for any reason. Extraction processes should also be non-invasive. Lake Vostok (Antarctica) is a possible terrestrial analog for exploring such non-invasive processes, especially in preparing for drilling through ice into potential aquifers. Because any given source of water will not likely be well understood, mission operations, at least in the beginning, should not depend on in-situ water sources until those sources are extremely well understood.

As a side note related to ISRU, it must be noted that infrastructure building, operations, and maintenance will all be sources of environmental disturbance that need to be addressed and minimized to the extent possible.

\section{Issue 4. Plant growth experiments and greenhouses}

Issue 4 includes two related but separate tasks. The first task is a science task to explore and understand through experiments how plant and microbial organisms from Earth interact with Mars material and how they can be grown at a Mars base location. The second task supports mission operations through the growing of food for the mission crew in greenhouse facilities located near or adjacent to the habitat. The second task depends upon at least some developments from the first task and involves more complex contamination questions. Although not addressed further here, the possible use of greenhouses to provide food products for Mars base crew support must be preceded by considerable development and critical overall mission planning decision, When the amount of provisions to be provided to the mission is determined (and total mission mass is being studied), what minimum amount of food products, if any on an initial human mission, can be assumed to be producible, with an appropriately high degree of assurance, for use during the surface stay and possibly during part of the return transit time?

\section{Plant growth inside the habitat}

Plant growth experiments and greenhouses inside the habitat should be treated consistently with habitat cleanliness requirements. If the experiments and greenhouses involve martian resources, they may have to be treated with extra cautions because novel organisms and new ecologies could emerge. Such organisms and ecologies could present unique risks to personnel health, particularly because they will be within the habitat. 


\section{Transitional experiments and missions}

Consideration of plant growth experiments and greenhouses raises the broader issue of assessing how to transition from biological experiments to food products for crew support on Mars. It will be prudent to implement precursor biological experiment missions prior to a human mission in order to assess the relevant biological issues, such as prospects for growth on or below the surface of Mars. Obtaining this knowledge early in the program development will allow for better mitigation of contamination risks.

An interesting transitional experiment issue will be to assess whether microbes or plants should be used first. Microbial experiments, both in reality and perception, may present a greater risk than plants. While perhaps unlikely based on our present understanding of Mars (e.g., lack of liquid water), microbes may be able to proliferate and spread in the environment in a way that a few contained plants would not. Keeping this in mind, an example of a possible transitional sequence might be: (1) axenic plants (i.e., plants without microbes), (2) small microbial experiments, (3) microbial ecosystems, (4) mini-greenhouse (highly contained, low biomass,), (5) larger greenhouses, and finally, (6) advanced life-support (e.g., significant plant production/growth, perhaps in the form of a large greenhouse).

Any such transitional biological experiments should be implemented consistent with planetary protection policies.

\section{Dispersal research is critical}

Dispersal research is critical here, as with many other scenario considerations. It is emphasized here because considerations of dispersion could result in important planetary protection distinctions regarding transitional biological experiments. For example, it may be determined that dispersal effects from any one of the above transitional steps are more or less than the others, suggesting what should be done first and/or what should not be done at all.

An example of a possible dispersal distinction that will likely have practical mission design and operations implications may be that a highly contained, low biomass plant growth experiment may not result in unacceptable dispersal of contamination versus a much larger, less controlled greenhouse which may disperse contamination over greater distances and in greater densities. Accordingly, dispersal effects of each of the above kinds of experiments/missions should be studied in terms of the potential for spread into the immediate local environment, the wider local area, regionally, globally, and to subsurface environments.

\section{Greenhouses and their operation}

A greenhouse would basically need to be treated as a habitat, but with consideration of the following key differences that could present additional planetary protection challenges: (1) martian soil/regolith would have to be sterilized and contained, (2) monitoring of emergent organisms and ecologies and associated leakage, keeping in mind the significant role of possible mutations, (3) outgassing of modified or novel gases, (4) leakage of liquid solutions, (5) greenhouse maintenance will likely be challenging and timeconsuming for many of the above reasons and more. Automation could help mitigate contamination risks associated with such maintenance - e.g., by reducing the amount of airlock activation needed by base personnel. Connecting the greenhouse to the habitat could also mitigate risks by minimizing outside activity and air-lock activation. 


\section{Related Earth-based research}

Clearly, there should be a strong emphasis on conducting as much relevant Earth-based research as possible prior to conducting transitional biological experiments on Mars. This would include simulating the martian environment to the best of our ability in both the laboratory and in computer models, and also attempts to study the dynamics of quite different "intersecting/interacting" ecosystems, again using both the laboratory and computer modes.

Plant growth experiments and any greenhouse utilization during an initial human mission to Mars also would be greatly assisted by relevant information on plant and microbe response to martian material that might be obtained from possible transition robotic missions, including experiments using MSR (Mars sample return) materials plus plants in very carefully controlled and sealed conditions.

\section{Issue 5. Subsurface Sampling}

\section{Sampling in the range of 10 meter depth}

For subsurface penetration in the 10 meter range, robotic sampling approaches should be used to minimize contamination of the surface site footprint and the subsurface environment.

\section{Sampling in the range of a kilometer depth}

Kilometer-range subsurface sampling likely will require the involvement of humans; however, human contact should be minimized. Remote subsurface analysis should be done first, if possible, and techniques for implementing this need to be a research a development priority.

Because cross-contamination of surface and subsurface environments must be avoided, aseptic penetration methods (e.g., cleaning and isolating the drill) should be used. Fluids could present a unique contamination challenge, which suggests the general need for research to investigate minimally invasive subsurface penetration methods. New technologies need to be assessed from a planetary protection perspective, especially since large samples may be needed. Some examples to consider are sterilized heat drilling, a mole approach, and multi-directional drilling. There is much literature describing alternative techniques and emerging technologies that needs to be considered.

\section{Equipment transport}

Cleanliness issues regarding transport of equipment to and from subsurface penetration sites are no different than those associated with distant collection as noted for Issue 1.

\section{Site documentation}

Detailed records of explored and sampled sites and appropriate environmental activities such as backfilling at subsurface sampling sites should be a priority to ensure the mitigation of environmental impacts. This holds for both scenarios 1 (distant surface sample collection) and 2 (sample analysis).

\section{Sampling of water versus permafrost versus regolith}

Planetary protection distinctions for subsurface water versus permafrost versus regolith will not be relevant for a near-term human mission because we will not likely have enough knowledge to make the scientific and operational distinctions, unless a substantial amount of precursor science data via robotic 
missions is obtained. However, such distinctions may be relevant as more is learned about the martian subsurface environment.

\section{Issue 6: What do you do if and when you find life?}

\section{The appropriate actions are scenario dependent}

The appropriate response to finding substantial evidence of life will likely be highly scenario dependent, and this topic could and should be the theme of a workshop of its own. Some examples of the scenario dependence are:

1. Current status of the life - alive at present or evidence of past life,

2. Form of the life - primitive or more developed, similar to any known Earth life (virus, bacteria, extremophiles, or other) or fundamentally different,

3. Biohazard level - possible level of toxicity, replication activity, potential for interaction with Earth life forms, other characterizations of active vs. benign, which are not likely to be easy to determine quickly,

4. Location - found in the near or deep subsurface or at or very near the surface (including via venting and/or flow processes)? How near to the base habitat - remote or unexpectedly nearby?

5. Extensiveness of the life-form - e.g., how much, how distributed, only one or multiple forms?

6. Temporal dependence - when in the mission cycle does the discovery occur?

7. Location of discovery - discovery outside the lab or in an on-site or remote lab? During the Mars human mission or during a returned sample examination in a lab on Earth?

\section{Procedures to be followed}

Clearly, pre-established procedures for foreseeable possible conditions should be followed, which raises the need for establishing such procedures ahead of time to the greatest extent possible. As noted above, establishing adequately broad and comprehensive procedures will require much thought since the conditions of interest appear to be highly scenario dependent with many unknowns and possible scenarios to consider. Establishment of these procedures should involve a broad community of international experts across a broad cross-section of disciplines. Procedures should include appropriate actions to be taken if unexpected conditions are encountered, which may then trigger a quickly convened group to formulate additional case-dependent procedures.

As an example, for a field discovery of life, high-level procedural guidelines might be:

1. Initially, do not touch or further disturb the life form and leave it where it is,

2. Perform non-invasive analysis to assess its nature - e.g., the first logical step would be to noninvasively determine if it is terrestrial contamination or martian in origin,

3. Set up a non-invasive monitoring station with several capabilities - e.g., visual monitoring at various magnifications could give much information and be easy to implement, 
4. Perhaps sample nearby area(s) to obtain proximal community/ecological context, and

5. Constantly reassess procedures based on new knowledge.

\section{Broader issues associated with the detection of martian life}

Planetary protection can be interpreted to include a quite broad range of considerations. Practical constraints kept this Workshop focused primarily on maintaining sample integrity for primarily scientific reasons and possible back contamination issues. But there are broader societal issues, such as other forms of value associated with Mars and possible life forms, as well as long-term environmental and ecological impacts to Mars, all of which ultimately involve difficult scientific and ethical questions that cannot be addressed in isolation. The discovery of extraterrestrial life would be an important moment for the human species. Thus, under broader interpretations of planetary protection, consideration of issues regarding what we should do once life is discovered should involve individuals from many segments of society. How we implement planetary protections considerations for our first mission to another world could set the tone for our prevailing attitudes toward subsequent exploration and possible long-term settlement.

\section{Workshop Group WG4B - Operations II: "Enabling a Safe, Productive Human Presence in the Exploration of Mars"}

Same Charge as WG4A: To explore several operation issues including habitat design, support and exploration equipment, and base operating procedures consistent with forward and back contamination control. A more specific assignment made at the Workshop was for the two "Operations" groups to include consideration of the following six scenarios for issues of back and forward contamination:

1. Distant surface sample collection

2. Sample analysis

3. ISRU (In situ resource utilization at the base)

4. Plant growth experiments and greenhouses

5. Subsurface sampling (both $10 \mathrm{~m}$ and $1 \mathrm{~km}$ depth)

6. What do you do if and when you find life?

\section{Working Group Members}

$\begin{array}{lll}\text { Brenda Ward - Rapporteur } & \text { Todd Stevens - Reporter } & \\ \text { Judy Allton } & \text { Charles Barnes } & \text { John Charles } \\ \text { Dean Eppler } & \text { Frank Grunthaner } & \text { Kent Joosten } \\ \text { Fred Rainey } & \text { Brent Sherwood } & \text { Perry Stabekis } \\ \text { Russell Vreeland } & \text { Robert Zimmerman } & \\ \text { Students - Lee Criswell, Melinda Miller, Howard Perko } & \end{array}$




\section{Preliminary Comments}

This second working group addressing "Enabling a Safe Productive Human Presence in the Exploration of Mars" organized their discussions and report on examining each of the assigned scenarios with respect to specific forward and back contamination concerns arising with the scenario. This group devoted considerable attention to the fifth issue, Subsurface Sampling, and much less to Issue 4 concerning plant growth experiments and greenhouses. Thus, the reports of the two parallel working groups complement each other quite well.

\section{Working Group Report: Operations II (WG4B)}

The report of Operations II (WG4B) addresses the same five issues in the same order as Operations I (WG4A). Operations II choose to specifically address forward contamination and back contamination considerations and concerns for each of these issues and to give more emphasis to the surface and subsurface sampling topics. This group also chose to express some of their observations and concerns in the form of questions.

\section{Issue 1. Distant surface sample collection}

Distant sample collection was defined to mean sampling away from the immediate vicinity of the habitat through the use of rovers, robots, and humans for locations where robots cannot operate easily or effectively.

\section{Forward contamination - Issue 1}

Break the chain of contact from the Earth habitat zone to the pristine martian environment.

The transition of equipment and personnel from the human-affected zone to zones of interest involves more than transportation and must be managed. An example of an additional need might include decontamination of exterior surfaces at a perimeter check point.

Cross-contamination between samples and between sampling sites are to be avoided. This may require means for cleaning and sterilization of tools between sampling events, use of multiple tools, etc. (See related problems listed under "back contamination"). To maintain scientific information, samples should be protected to avoid unintended sterilization during sampling or transit.

The integrity of possible in situ life forms should be protected. For instance, a martian site should not be destroyed in the course of sampling it. (Note that the group expanded definition of forward "contamination" to include physical damage imposed upon the martian environment).

\section{Back contamination - Issue 1}

Break the chain of contact between potential martian habitats and the human habitation zone. Samples should be contained at the point of acquisition. In situ evaluation of samples, if and when possible, might provide a valuable tool by reducing the need to obtain samples and/or by allowing earlier assessment and containment of more selectively chosen samples.

The possible recovery of sampling equipment and its reuse at another site involves the possible contamination of the second site by material from the first. Reuse of sampling equipment requires means 
for cleaning and sterilizing this equipment in the field. How will equipment be recovered and cleaned in the case of equipment malfunction?

The movement (if any) of equipment from a zone of interest back to the base habitat zone or to the next zone of interest should be managed to prevent contamination.

If humans operate within remote potentially contaminated zones, how will decontamination be done? How would an incapacitated human be rescued and recovered?

\section{Issue 2. Sample Analysis}

This report is a summary of the topics included in the wide-ranging group discussion on many aspects of sample analysis.

\section{Forward contamination - Issue 2}

Some means is needed to detect forward contamination (there was some disagreement among the group on the degree of need, on what this might entail, and on what it would require). The primary purpose of this QA/QC (quality assurance/quality control) program would be to identify and/or prevent false-positive results and thus avoid the unnecessary triggering of PP contingencies. Examples might include control experiments, physical or chemical tracers, witness coupons, bioload inventories, etc.

One cannot simply assume that all analysis procedures will always be perfectly adequate or perfectly executed. If analysis data are not collected in real time, it may be impossible to reconstruct individual analysis details later and this might make it impossible to clearly identify false-positive results.

\section{Back contamination - Issue 2}

The following observation from an earlier workshop session: "Acquisition of samples must be in a manner that isolates samples from crew and habitat. Sample storage, handling and screening should be done under containment, isolated from the habitat, etc." was reinforced and noted to include sample analysis and analysis equipment contacting the sample.

\section{Issue 3. ISRU at Base}

The potential scale of in situ resource utilization (ISRU) at and near the martian base may involve issues of controlling contamination for larger volumes of material than for any other activity. The volume of material that might be involved in ISRU could be at least an order of magnitude larger than from any sampling.

\section{Forward contamination - Issue 3}

Industrial processes may have significant impacts on the surrounding martian environment. These processes and the associated equipment and facilities should be designed to minimize these impacts. On site, their effects should be monitored and the boundaries of an Earth-contaminated zone may need to be adjusted outward as the ISRU operations progress.

Some processes may create potentially hazardous by-products, such as corrosives (especially during the processing of water). The disposition of any hazardous by-products should be monitored and, if necessary, mitigated. Forward contamination may be more problematic in utilization of water resources. 
Ensure that industrial processes do not affect regional or planetary ecology remote from the industrial zone. Effects of ISRU should be limited to a carefully selected and clearly designated zone.

How much impact on the site is acceptable? What are the relevant criteria for such a decision?

\section{Back contamination - Issue 3}

Before any native materials are introduced into the habitat, they should either be thoroughly screened for potential life forms and/or biohazards or the material should be sterilized.

The scale of potential processes may present a problem of magnitude (e.g., mining large volumes of ice or ice-bearing material that might contain small numbers of native organisms).

The introduction of potentially hazardous ISRU by-products into the habitat, or of corrosive by-products to its exterior should be avoided.

\section{Issue 4A. Plant growth experiments internal to a habitat, no ISRU}

\section{Forward contamination - Issue $4 A$}

An increased terrestrial bioload associated with contained plant growth may increase the possibility of forward contamination, and an increased and more complex terrestrial bioload in the habitat may complicate monitoring for false positives.

\section{Back contamination - Issue $4 A$}

No significant back contamination issues were identified for contained plant growth away from contact with martian material.

\section{Issue 4B. Plant growth experiments using martian materials (may be contiguous with habitat)}

\section{Forward contamination - Issue $4 B$}

Assuming a contained experiment is not contiguous with the habitat, the possible mixing of a terrestrial ecosystem with a potential martian ecosystem could potentially create a new "third ecology." Even when the contained experiment is not contiguous with the habitat, it may be prudent to avoid contaminating either Earth (including the habitat) or Mars with the potential new entity.

\section{Back contamination - Issue $4 B$}

Assuming an experiment or greenhouse contiguous with the habitat, the biosafety of all martian materials introduced into the habitat should be assured before their introduction. This might involve ensuring that the material contains no living organisms (by analysis) or by sterilizing the material.

\section{Issue 5. Subsurface sampling}

Subsurface sampling may present fundamentally different problems than other types of exploration because the sampling target cannot be directly observed. 


\section{Forward contamination - Issue 5}

"Aseptic" (free from pathogenic microorganisms) drilling may be easier on Mars than on Earth because the surface layer is likely to be sterile and therefore not a source of general contamination.

Forward contamination should be reduced by sterilizing sampling tools and all down-hole equipment. If the technology used requires repeated sampling, removal of down-hole equipment, and reentry of the hole, then some means for onsite re-sterilization of tools may be needed between each sampling.

Steps to prevent cross-contamination of a sample with material from upper strata are needed, with details dependent upon the drilling and sampling procedures. For example, the hole may need to be cased or sample extracted through an annular wire-line. Reentry of holes for subsequent core sample would increase concerns about possible cross-contamination.

The outermost portion of cores is most likely to be contaminated with material from the surface and the upper portion of the hole. It is best to return the core to a nearby sample-processing glovebox as soon as possible for paring and subsampling in order to prevent infiltration of contaminants. Tracer methods may potentially aid in verifying pristine samples and identifying false-positive samples.

\section{Back contamination - Issue 5}

Most deeper drilling technologies utilize circulating drilling fluids to return cuttings to the surface and to cool and lubricate the bit. The most convenient drilling fluid may be compressed martian atmosphere (although this fluid would dry any water out of the formation material coming into contact with it). Regardless of the drilling technology used, if water or ice is encountered, it too will form slurry that circulates in the hole. The fluids returning to the surface and their entrained cuttings present a serious potential for back contamination.

Means for avoiding contamination of the equipment and personnel by the returning fluids and cuttings are needed. Possible examples include containment or controlled dispersal of this material. (Note that the cuttings may also constitute useful samples).

If a pressurized zone were to be encountered or created by drilling operations, uncontrolled ejection of potentially contaminated material could occur. Systems should be robust enough to contain or divert blow-outs.

It is the nature of all but the most limited drilling that human intervention is regularly needed to repair and maintain the equipments. Means should be devised so that astronauts can safely approach and manipulate the equipment without introducing potentially contaminated material into either the drilling site or, upon their return, to the human habitat. This may require a new direction in drilling technology development. Examples might include containment of drilling fluids and means for cleaning and sterilizing the space suit and tools.

Can real-time sensors be used to assist in planetary protection measures and sampling? For example, monitoring for moisture in returned drilling fluids might indicate the drilling is approaching a possible microbial habitat.

There may be an increased risk of events such as a suit breach or astronaut injury because of the proximity of personnel to industrial-scale drilling and the associated machinery, along with risks of exposure to or release of potentially contaminated material. 


\section{Issue 6: What do you do if and when you find life?}

A diversity of opinion among the working group members concerning the appropriate responses to any discovery of martian life was evident from the several sets of notes used to compile the composite response given below. In general, the discussions centered on follow-up actions at the discovery site and on management of communications of the discovery to the general public.

\section{Follow up analysis and management of the discovery site}

Astronaut explorers can be trained to recognize additional sampling sites that may also have life. This additional sampling can help establish a site context using an iterative approach to help define what site characteristics are associated with the life.

A distinctive site marker should be placed at the discovery location so it can be later avoided or resampled, as appropriate.

A considerable diversity of opinion surfaced on what would be an appropriate follow-up once martian life is discovered. Options supported ranged from (1) leave it in place and conduct no invasive study to (2) study it on Mars, to (3) bring it back to Earth for detailed study. Support was voiced for a policy that confirmed martian life should never be brought back to Earth. A counter observation was that if planetary protection actions are done correctly, where martian life might be studied should not matter, at least by scientific considerations.

Depending upon the maturity of planetary protection policies at the time martian life would be found, once confirmation is reached that life has been found, exploration may need to cease and new more specific planetary protection protocols be developed, depending upon the general characteristics of the life forms found, before exploration activities are resumed.

Field (including in situ) analysis of martian life may offer a controlled environment and facilitate contained disposable experiments that should help in resolving issues of whether we have actually found life and whether or not it can be returned to Earth safely. However, field analyses may have limited analytical capabilities. There was some dissension on the probable usefulness and advisability of field analyses.

In all cases, direct exposure of astronauts to any samples found to contain or suspected to contain life must be avoided.

\section{Policy and public relations issues}

Psychological preparation of the general public to the possible discovery of martian life should be addressed. Politics and public perception must be considered all along as plans are made for missions to Mars. The Mars missions need the support of excellent and effective communications giving people an understanding of why we are going there and what is being done to protect Earth from possibly being contaminated by organisms that might be discovered on Mars. What the mission might find, the possible impacts to Earth and the control of the associated risks need to be generally understood based on a realistic science-based assessment, rather than on the entertainment-based themes of Hollywood movies and science fiction publications familiar to many or most of the general public.

Even though from a planetary protection viewpoint, absolute containment, both forward and backward, is the goal in mission design, technology limits, contingencies and funding cuts could compromise that containment unless everyone understands the importance of maintaining absolute containment. 
Conversely, can the risks of back contamination be reduced to a level low enough that the public will accept the very small but finite risk associated with achieving the scientific and philosophical benefits associated with the possible discover and analysis of martian life? What risk level for each possible hazard and consequence combination is acceptable, and how are these maximum tolerable risk levels to be set?

The mission personnel may need to being prepared, as much as possible, to study any martian life found during the mission either in situ or in a facility located on Mars because of what may be an intense public response and political ramifications when it is disclosed that the bringing back of Mars life to Earth is planned and/or will take place. Some mention was made of polling public opinion after any discovery of martian life but before it would be brought to Earth.

Because communication with and input from the public is vital, a workshop devoted to the issue of how the public perceptions will influence future Mars missions re planetary protection and to planning of these interactions with the various publics involved (citizens, decision makers, international community, etc) is recommended. 


\section{Plenary Discussions and Recommendations on Operations}

The Workshop concluded with an early afternoon plenary session on Saturday, June 23, which was divided into two parts. First, the two working groups on Operations II and I presented their findings, followed by overall group discussion on operations, including on the topic of "what do you do if and when you find life on Mars?" After a brief report on planned future European missions to Mars given by Daniel Prieur, the plenary group began its final scheduled task of formulating overall conclusions and recommendations. The following section summarizes input from all workshop participants assembled in plenary session on the six operational scenarios assigned.

\section{Operations enabling a safe, productive human presence in the exploration of Mars}

The assembled workshop group spent more time on some of the six operational questions than others, as is reflected in the comments recorded. The major points given particular emphasis in the reports brought to the plenary session or arising first during the ensuing broad-ranging discussions included, in the order they were made for each issue:

\section{Issue 1. Distant surface sample collection}

- All equipment and basically everything used in sampling should be cleanable to meet standards yet to be defined in order to prevent their being the source of cross contamination.

- Routes and paths to sites of interest need to be designed to minimize adverse impacts to both the region through which they pass and especially to any intermediate areas of high biological interest.

- New technology is needed to minimize forward contamination from space suit outgassing and leaks and from various equipment and operational procedures.

- Information is needed on how well the techniques of gas sterilization will work on Mars.

- More information and protocols are needed on how to manage both forward and back contamination issues when humans venture into locations where robots cannot be sent or need to be used in combination with humans.

- More information is needed on how to best clean and sterilize tools/equipment between samples, with special attention on avoiding cross contaminations, within the resource constraints of an early human mission to Mars.

- It is necessary to break the chain of possible contamination between the samples and the occupied habitat.

- In situ evaluation of samples should be done to the extent possible to provide needed information, as many potential contamination issues can be avoided if the sample does not need to be further handled.

- Guidelines need to be defined on what response should be made to a mishap such as a broken sampler, malfunctioning equipment, accident or other abnormal condition.

- A human presence will probably be required during sampling to obtain full site context information, to interpret conditions and to protect endangered life forms if any are found.

- The phrase "cleaned to TBD specifications" raises the question of who will determine these "tobe-determined specifications," when will this be done, and how demanding these standards will be.

Issue 2. Sample analysis

- Containment redundancies are necessary during sample preparation and analysis. 
- Spill containment and cleanup policies and procedures should be well defined, tested, and in place well before the first arrival of any martian samples to the laboratory, whether on Mars or a sample-return facility on Earth.

\section{Issue 3. ISRU (In Situ Resource Utilization) at the base}

- ISRU likely will present the need to deal with contamination issues for large volumes of materials.

- ISRU operations should be conducted so they do not affect regional or planetary ecology away from the ISRU zone.

- Equipment and processes related to ISRU must minimize any undesirable introduction of hazardous materials or by-products into the planetary environment.

- When it is necessary to vent gases, these gases should be filtered before release into the environment.

- Ground/surface disturbances should be kept to a minimum, as should be the production of dust and piling of fine, loose material that could be picked up and transported by the martian winds.

- Lake Vostok, located far under the Antarctic ice surface, may provide an excellent venue for modeling how to treat subsurface water discovered on Mars (and also as a site for study of deep drilling, Issue 5).

- Including ISRU on the first mission may not be advisable, or should be very limited because of contamination concerns.

- Contaminant/dust dispersion paths and mechanisms should be studied and understood before ISRU operations are undertaken.

- Plan for the worst-case scenario - e.g., release of radioactive isotopes, toxic materials.

\section{$\underline{\text { Issue 4. Plant growth using some martian resources }}$}

- If martian materials are used within the habitat and/or greenhouse, all concerns noted for ISRU also apply.

- The contact of plants and associated organisms from Earth with the Mars environment could conceivably result in a third ecology, one having unpredictable properties.

- The transition from plant experiments to the utilization of plants to provide food needs careful management, as the necessary direct human contact with food grown in contact with martian resources greatly increases contamination concerns.

- The processes of growing, harvesting, and processing of plants or other organisms (i.e., fungi, algae) for food, either as applied research or to provide food for the humans, must be carefully planned and conducted to avoid possible contamination to the astronauts at any time.

- The dispersion of gases previously in contact with plant and/or microbial life and other contaminants that possibly would be released from the greenhouse into the Mars environment also requires extensive study.

- As with sampling and sample handling, containment redundancies may be necessary.

- Spill containment and cleanup policies and procedures should also be in place in greenhouses, as with sample handling, especially when ISRU is part of the greenhouse operation.

- Human access to greenhouses and the need for human intervention under normal and abnormal conditions within the greenhouse must be well defined, regulated, and related to the hazard level present, including consideration of the level of ISRU use.

- There is a need for Earth/based research on particulates and aerosols and their dispersion behaviors in general and in the conditions to be encountered on Mars. 


\section{Issue 5. Deep drilling}

- New technology is needed to effectively carry out drilling operations in remote sites, especially considering that they will be conducted in very demanding conditions and may be to depths requiring quite heavy equipment and large power needs. The demands of preventing cross contamination and the probably inapplicability of the usual water-based drilling fluids almost always used on Earth both comprise very significant additional design conditions.

- To help prevent contamination from drilling operations, including cross contamination among formations at different depths, a plan for sealing/filling of drilled holes and site cleanup and the use of such a plan is necessary.

\section{Issue 6. What if life is detected on Mars?}

- This topic is deemed very worthy of a separate workshop dedicated to this one subject.

- It must be assumed that martian samples contain life until it can be proven otherwise.

- If life is found, determining the extent of the presence of that life is a logical goal, although this may be operationally difficult to do.

- The appropriate response by a human mission on Mars to the discovery of life will significantly depend on when in the mission cycle the discovery is made and on several other factors.

- What should be done if martian life is found to be present in the astronaut habitat, perhaps due to the breakdown of contamination protections?

- Non-invasive monitoring and assessment techniques are very desirable for any study of suspected or determined martian life, including for its characterization and to help define its extent.

- A human mission to Mars needs to bring along useful equipment and experiments to help determine the characteristics of any life that is found, in part to minimize the need to bring such life back to Earth for study.

- Protocols need to be tailored to the possible general nature of the organisms, if that is possible or practical.

- Psychological preparation of the public for the possible discovery of life on another planet is needed. The exploration and scientific community needs to be very proactive, starting now, to address public communication opportunities and issues. Along this line, a Workshop dedicated to planning these tasks with input from sociologists, theologians, politicians, other policy makers, and with international participants is recommended. 


\section{Overall Workshop Conclusions and Recommendations}

This was an initial effort to address a broad and future-oriented topic. Thus, the overall conclusions and recommendations from the Workshop are quite general, and are expected to be further honed over time. Consistent with the exploratory and brainstorming environment fostered at the Workshop, and the inclusion of a wide diversity of professional viewpoints and expertise, this initial Workshop was not intended to be comprehensive of all possible PP concerns, nor to provide rankings or priorities of planetary protection issues. The conclusion that follow, therefore, are intended to serve as a start and springboard for later discussions, workshops and actions.

\section{Conclusions}

1. Planetary protection measures must be considered early in the planning for the first venturing of humans to Mars. Meeting these requirements to eliminate, mitigate or minimize risks of contamination is a mission necessity. Indeed, planetary protection issues must be a part of such a mission from the initial stages of mission planning and hardware design, whether robotic or human missions, when going to sites that could conceivable harbor life in any form. In general, most planetary protection needs are consistent with the requirements of properly conducted science, prudent engineering design, general astronaut/crew health, identification and control of project-related risks, and overall planning for project success. Crew safety, integrity of planetary protection, and overall mission success are closely related overall goals.

2. Including humans in a planetary exploration mission very significantly increases the challenges of providing adequate planetary protection over a purely robotic mission for several reasonsespecially including the fact that humans nurture a complex, internal and external microbial community related to digestion, and many other human functions. Planetary protection considerations include the impossibility of sterilizing humans, the continuation of life processes that require breathing oxygen, water and food and which produce respiration, digestive and liquid waste products, and the multitude of ways in which martian contaminants might affect human health and performance.

3. Humans have unique capabilities in recognizing likely sites that might harbor life, in deciding which sites should be sampled and how, in carrying out that sampling, and in making quick assessments and evaluations when unexpected conditions arise. As the use of robotics avoids or reduces many of the contamination concerns associated with suited astronauts and associated support equipment at the sampling site, routine and repetitive tasks should be assigned to robotics whenever practical. The division of responsibilities and cooperation between robots and either remote or accompanying humans is presently difficult to define, as this division will be dependent upon the capabilities of robotics at the time the mission details are being planned, as well as the familiarity of the mission planners and the astronauts with such cooperative operations.

4. All mission operations should include the stepwise relaxation of the isolation of humans from contact with materials of martian origin for planetary protection purposes. Astronauts should not be exposed to Mars unless and until those materials have been shown to be benign and free of any life forms, a classification which may be difficult to verify during an initial human mission. Steps should be taken for sample control, sample isolation, and to break the chain of contact between martian material and the extended environment of Earth, ranging from the astronauts, themselves, while on Mars to the Earth, itself, via sample return. Sample-return safety for robotic precursor 
missions is being addressed by the ongoing preparation of biohazard-related protocols, and it is expected that these protocols will be compatible with those needed later for human missions.

5. A number of actions are needed in order to reach a more complete understanding of the general and localized environments of Mars (e.g., local region around a habitat, sampling sites, base habitat, laboratories, greenhouses, etc.) and other exposure considerations necessary for proper mission planning. This improved knowledge will impact technology and hardware designs for the planned human mission. The environment in which the first human mission will operate needs to be known and understood as quickly as reasonably possible. Information from precursor robotic missions, both planned and future, should be conducted to provide many types of information, including general and specific site characterization and general characteristics of the martian dust in the global-scale dust storms. Although it is not thought likely that this dust will itself harbor martian life, the degree to which it might be a biohazard and/or be chemically reactive is very basic information that must be known, and which could be a key to the design of many features of a human exploration mission. Further study is also needed to understand the indigenous microbial load in enclosed human occupied environments, such as those entailed in a human mission to Mars, and on likely mutations among microbes in both enclosed and outdoor environments.

6. Further definition of a system describing martian locations according to special scientific interest and according to operational clearance status (level of contamination danger), such as suggested by Working Groups 1 and 3, is needed. These classification systems should be employed in formulating future planetary protection protocols, project planning, and mission operations. While locations of high scientific interest may be most attractive to a large segment of the science community, the first human mission base/habitat should itself be sited at a location of no inherent biological interest, although this site may be selected so that locations of biological interest may be reached by rovers and robotics from the initial base location.

7. Further attention must be given to long-term planetary protection requirements with respect to forward contamination and its possible interaction with surface features and disturbances on Mars. Possible cross-contamination of sites on Mars, including those reached during drilling and/or sampling operations, is among the planetary protection issues of importance. The crosscontamination phenomenon is quite analogous to the Lake Vostok (Antarctica) sampling questions now under discussion. There could be emerging issues regarding ecological developments on Mars associated with contact between Earth organisms and martian materials (either unintended through forward contamination or planned through ISRU projects), forming a "third ecology" concept, with presently unknown implications.

8. It is not easy to apply Earth-based definitions of life to possible martian life. Given recent discoveries on Earth of life with surprising characteristics, and thriving in severe environments thought not to be conducive to life (e.g., extremophiles living among deep sea thermal/volcanic features and life deep within caves), it is difficult to predict what might be the general nature and characteristics of any life found on Mars. How do we define life, and given the many possible forms, how will we be able to reliably detect and characterize this possible martian life of a presently unknown nature? To what degree do we know what we are looking for?

9. Improved technologies need be to developed in many areas in order to meet mission needs and PP requirements - these range from astronaut suit design to habitat life support systems to sampling and analysis equipment. Deep subsurface sampling operations now appear particularly problematic on Mars with respect to both operational and planetary protection issues, and given current and anticipated future technology. 
10. General human factors need to be folded in with planetary protection issues for a human mission to Mars. First, it is difficult to describe issues related to planetary protection separate from other human factors. Second, there can be significant interactions between planetary protection issues related to human health and both general health and psychological considerations. An example of one such notable interaction is possible physical effects from contaminants, including from martian dust, leading to debilitation and reduced performance in astronauts. This can lead to unintended actions which can in turn lead to mishaps, some which may have particularly serious consequences for planetary projection. Mission planners should be particularly vigilant to avoid overloading the mission astronauts with tasks, and should closely monitor actual and perceived astronaut physical and mental health conditions, since mistakes are much more likely when humans are tired and stressed, or overly-stressed.

11. The first human mission to Mars will face a significant level of risk, as it will involve a high degree of venturing into the unknown using complex transportation and life support systems. Because of the remoteness of Mars and the requirements of orbital mechanics, the mission faces long transit times to/from Mars, periods of dynamic flight conditions, and demanding flight navigation controls. Orbital mechanics also dictate the duration of the mission's stay on the martian surface - this duration ranging from about a month to over a year, depending upon the flight path option chosen, and the stay in the vicinity of Mars cannot be cut short if mission difficulties are encountered. Total mission times are in the range of about 2 to over $2-1 / 2$ years, with the return flight possibly being effectively a crew quarantine time (N.B. The potential for astronauts to develop health issues, unrelated to exposure to possible Mars organisms, during this period is likely higher than normal). Regardless of the development effort and the amount of component and prototype testing, the first human mission will be the initial test of the fully operational and complete system for its intended purposes. Planetary protection risks are among the many risks to be identified, defined and understood, evaluated, and then reduced, mitigated, or eliminated when possible to help assure mission success.

12. A primary question is whether a future human exploration mission to Mars can be accomplished in ways that avoid harmful cross contamination of that planet and Earth, consistent with international and domestic PP policies, while being scientifically productive. The Workshop participants generally concluded that it is conceptually possible to develop systems, exploratory approaches, and operations plans to enable a safe, productive human mission to the Mars surface, although planetary protection requirements will most definitely affect the design, operation, and cost of advanced life support, environmental and scientific systems for long duration human missions.

\section{$\underline{\text { Areas of needed research }}$}

In addition to the research needs entailed in several of the conclusions given above, the following research needs were specifically noted:

1. Research on the dispersion of dust and contaminants in general and specifically in the martian atmosphere is critically needed. Both general modeling capabilities and precursor mission information on the spatial dispersion and characteristics of martian dust transported by wind and other means is needed. How much of a biohazard martian dust presents and its degree of chemical reactivity is information which can be critical for human exploration mission design, and fairly early precursor missions should be employed to help provide dust characterization details. 
2. Future research will be needed on the potential impact of a variety of human support activities expected to be part of the operation of a martian base, including providing breathing oxygen, water and food supply, waste management, thermal control, and energy needs.

3. A significant research and development effort is needed to determine how robotics can best help conduct operations on Mars in a way consistent with PP concerns, both independently during precursor missions and in conjunction and cooperation with humans during later missions.

4. Improved space suit designs consistent with PP issues (such as use of effective filtration to assure no human-borne microorganisms are vented) and the demands of human activities on the martian surface, especially for activities conducted away from pressurized habitats and rovers, are needed.

5. Much research and development remains to be accomplished concerning the technology required for life detection and characterization, potential pathogen, biohazard, and toxicity detection, and the sensitivity and specificity of various tests, especially those needed to define and determine critical criteria such as "how clean is clean enough" and "how "alive" is indeed "alive" when determining if life has been found.

6. Formulation of a site classification system and a biological plausibility (i.e., the extent to which the site is thought conducive to the support of life) mapping format for both martian surface and subsurface conditions is needed, followed by the utilization of photographic, ground penetrating radar, and other remote sensing information. Any available returned sample analyses, and other information from precursor missions should be used to help in producing such maps. Particular maps are suggested to include areas of special scientific interest (including locations where water in a liquid form might exist), levels of contamination concern, and zoning for permissible human mission operations.

\section{Recommendations for future workshop topics}

Given the exploratory and general nature of the Workshop, it should not be surprising that a myriad of potential future workshop suggestions arose. Many topics directly related to the planetary protection issues discussed in the Workshop were identified as needing more consideration than was possible at this initial workshop. The priority topics generally fell into one of three categories.

The first group of topics concerns possible martian life, starting with how life is to be defined given that extraterrestrial life may be much different than life as it is known on Earth. How can such life be detected and characterized. Perhaps as least as important as life detection for planetary protection purposes is how can the absence of life from a given location or within a given sample be determined with a high level of certainty.

The second group dealt with how to better identify and characterize the many planetary protection issues and their coordination with base operations and scientific objectives. Only with more complete information can more detailed planetary protection protocols appropriate for future human exploratory missions to sites such as Mars be developed.

The third set addressed the many tasks and challenges that the human explorer will face in a remote, unfamiliar environment and his/her likely responses to these conditions. A workshop on general health, life support, work environment, psychological health, and other human factors, along with their interactions with planetary protection issues and human decisions and response to the implementation of 
the PP requirements was suggested. Such a workshop could be of great assistance in the understanding and subsequent planning of the human involvement in space exploration.

Topics requiring consideration in future workshops, perhaps as workshop sub-themes, included:

1. Training for the Mars mission crew members in microbiological principles and basic laboratory techniques, along with other technologies critical to planetary protection assurance. How much of this should be done? How significantly will such background information improve crew member performance and philosophical "buy-in" with the PP and overall safety concerns?

2. Many ethical issues arise with a human mission to Mars, especially if the mission detects life. Some are addressed by international laws and various protocols, others are not.

3. Although the Workshop addressed contamination issues primary related to sampling, sample return, ISRU activities, and other science-related activities, contamination considerations also apply to the day-to-day operation of the habitat, the handling of wastes, and the return of the human explorers to Earth, which involves also the return at least a re-entry vehicle and some equipment and life support systems.

The assembled group choose to give special attention to topics on quite broad issues in their recommendations for specific future workshop topics, rather than to begin to list possible worthwhile workshops more directed to the specific tasks, mission operations, and definition of planetary protection requirements topics noted above. This decision was partly based on an expectation that workshops on procedures and protocols, operational requirements, and other more mission-directed topics would more naturally develop as planning for the first human mission to Mars evolves.

The result was the recommendation of the following three potential workshops, with the first being the most often mentioned:

1. "If Life, Then What?" This workshop would address appropriate responses to the discovery of martian life, whether this discovery was in a robotic mission sample return mission or during the first or later human mission to Mars. Although emphasis would be on the scientific aspects of this question, some inclusion of other considerations (international law, ethics, public polity and politics, etc.) should also be included.

2. Astronaut/Explorer Health and Performance - General health issues for humans in a remote, unfamiliar environment, life support requirements, work environment, psychological and other human factors, along with their interaction with PP issues and general performance during an extended mission of many months to a few years, much in quite confining quarters, including travel times.

3. Communications with the Public - This workshop or series of two workshops would address; (a) the general public response to the detection of extraterrestrial life on Mars (or elsewhere away from Earth)-including their concerns, their perceptions and understanding of what forms this life might be, the likely response of the scientific, political, religious, international and other communities, and (b) the use of communications to prepare the public for the possible discovery of non-terrestrial life - what background information and project details are most wanted and needed by the general public, what degree of validation/assurance of a the finding of life or other major discovery is needed before a broad announcement is made, how to best involve the public in the exploratory process, and many related questions. 
These suggestions for future workshops are consistent with this Workshop being only an initial meeting addressing the exciting, complex, and challenging topic of the future human exploration of a remote site that might harbor life. Such human exploration missions, if properly planned and conducted, will be key in helping provide answers to a long list of questions of high interest to humankind, including "Are we alone in our Universe?" 


\section{References}

CDC-NIH (Centers for Disease Control - National Institutes of Health), 1999. Biosafety in Microbiological Laboratories, $4^{\text {th }}$ edition, U. S. Government Printing Office, Washington, D.C.

NASA, 2001. The Mars Surface Mission: A Description of Human and Robotic Surface Activities (NASA TP-2001-20937), NASA, Washington, D.C.

National Research Council (NRC), 1992. Biological Contamination of Mars: Issues and Recommendations. National Academy Press, Washington, D.C. <www.nap.edu>.

National Research Council (NRC), 1997. Mars Sample Return: Issues and Recommendations.

National Academy Press, Washington, D.C. <www.nap.edu>.

Puleo, J. R.; Fields, N. D.; Bergstrom, S. L.; Oxborrow, G. S.; Stabekis, P. D.; and Koukol, R. C.: Microbiological Profiles of the Viking Spacecraft. Applied and Environmental Microbiology, February 1977, pp. 379-84.

Race, M. S.; DeVincenzi, D. L.; Rummel, J. D.; and Acevedo, S. E. (eds.): 2002. Mars Sample Handling Protocol Workshop Series, Workshop 4 Final Report, NASA CP-2002-211841, Washington, D.C. Available online at: <planetaryprotection.nasa.gov>.

Race, M. S.; Criswell, M. E.; and Rummel, J. D.: 2003. Planetary Protection Issues in the Human Exploration of Mars. Paper Number 2003-01-2523, International Conference on Environmental Systems (ICES), Vancouver, B.C., July 2003. Available at <www.sae.com>.

Rummel, J. D.; Race, M. S.; DeVincenzi, D. L.; Schad, P. J.; Stabekis, P. D.; Viso, M.; and Acevedo, S. E. (editors): 2002. A Draft Test Protocol for Detecting Possible Biohazards in Martian Samples Returned to Earth. NASA/CP-2002-211842, Washington, D.C.

United Nations, 1967. Treaty on Principles Governing the Activities of States in the Exploration and Use of Outer Space, including the Moon and Other Celestial Bodies. U.N. Doc. A/RES/2222(XXI); TIAS No. 6347, New York, USA. January 1967. 


\section{Appendix A: Workshop Venue and Agenda}

\section{Conference Venue}

The Pingree Park facilities are at the Mountain Campus of Colorado State University. Pingree Park is located in a mountain valley close to the northeast corner of Rocky Mountain National Park at about 9000 -ft elevation. It is located about 50 miles northwest of Fort Collins and about three hours travel time from Denver International Airport.

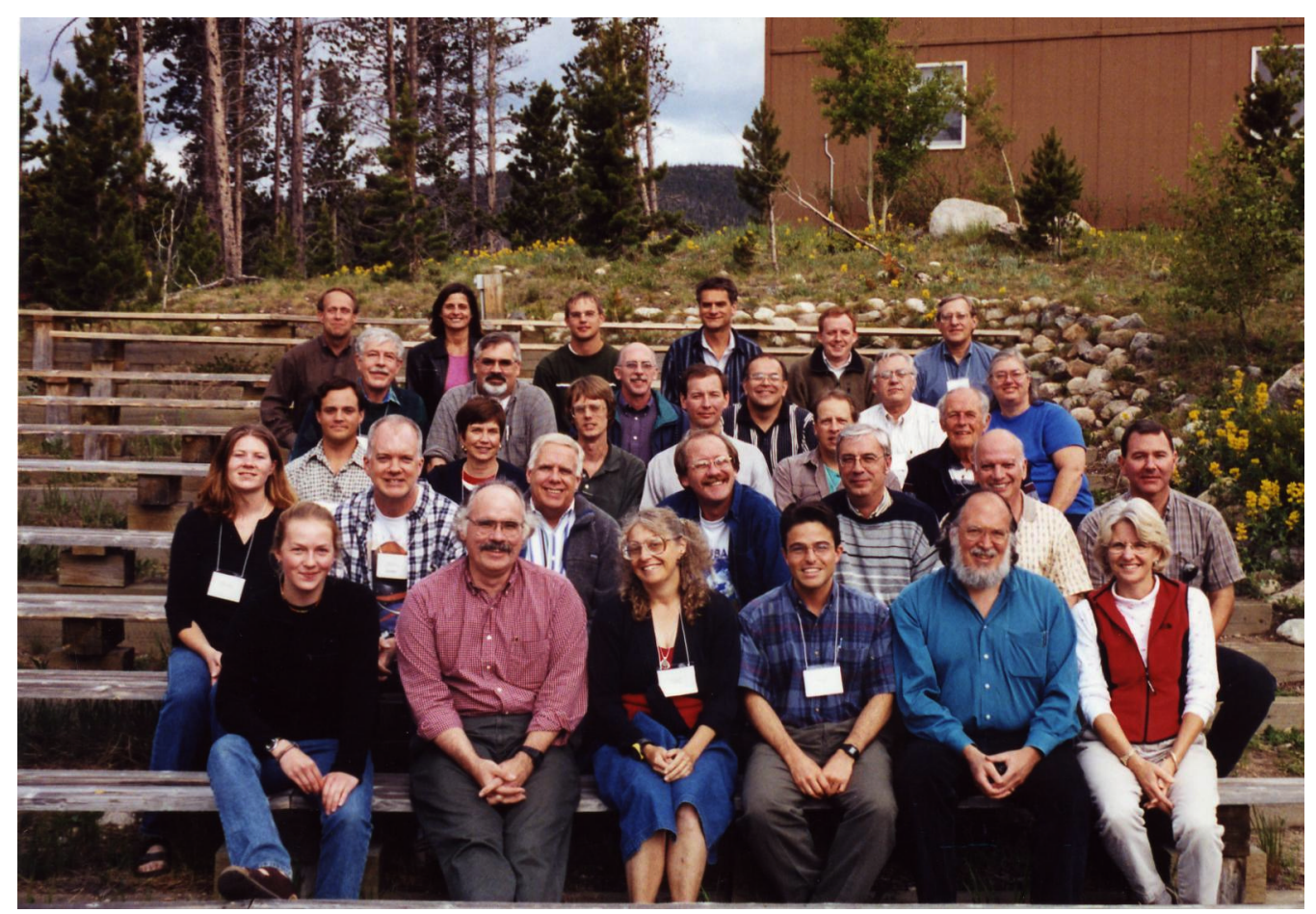

Top left to right: John Rummel, Brenda Ward, Jeff Carlson, Chris McKay, Fred Rainey, Dean Henniger Row 2: Marvin Chriswell, John Battista, Charles Barnes, Russell Vreeland, Frank Grunthaner, Lee Criswell

Row 3 Howard Perko, Judy Allton, Richard Fullerton, Mark Kliss, Todd Stevens, Robert Phillips Row 4: Melinda Miller, John Charles, Don Eppler, Kent Joosten, Daniel Prieur, Perry Stabekis, Dale Anderson

Row 5: Katie Hams, Steven Hoffman, Penny Boston, Mark Lupisella, Robert Zimmerman, Margaret Race

(missing: Gary Martin, Brent Sherwood, Diana Wall, Albert Yen and Marilee Rowe) 


\section{Meeting Schedule/Agenda}

Wednesday, June 20, 2001

7:30-8:30 p.m. Informal Reception

Tuesday, June 21, 2001

7:30-8:30 a.m. Breakfast, informal at Dining Hall

9:00 - noon Workshop Introductory Session

Introductions and workshop charge, workshop objective, organization Background presentations

Why send humans?

What is life and how can it be detected?

NASA plans and possible timetables

Capabilities and needs of humans in extended space missions

The martian environment

Planetary protection requirements

Forward and back contamination - current status

Noon-1:00 p.m. Lunch, informal at Dining Hall

1:15-4:15 p.m. Individual Working Group Meetings

WG1 - Protecting Mars \& Science

WG2 - Protecting Human Health

WG3 - Protecting Earth

Tasks: Identify major concerns and challenges, constraints and barriers

Working group organization, start of working group report outline

\section{4:30-5:30 p.m. Brief preliminary report from each Working Group to} the assembled Workshop

Evening

\section{Dinner, informal at Dining Hall}

Followed by possible informal meetings or work sessions of the first day working groups, at their option 
Friday, June 22, 2001

7:00-8:15 a.m. Breakfast, informal at Dining Hall

8:30-noon Individual Working Group Meeting

WG1 - Protecting Mars \& Science

WG2 - Protecting Human Health

WG3 - Protecting Earth

Tasks: Obtain/define relevant information and knowledge

Work toward identifying resources, solutions and recommendations Identify and consider interactions with other working groups

Complete outline of working group report and assign tasks

Noon-1:00 p.m. Lunch, informal at Dining Hall

1:00-2:15 p.m. Plenary Session - Individual Working Group Reports and Discussion

Reports from WG1, WG2, and WG3

Group discussion on each working group report

Reformulation of participants into new Working Groups:

WG4A- Operations I, and WG4B - Operations II

\section{2:30-5:15 p.m. Individual Working Group Meetings}

WG4A-Operations I

WG4B - Operations II

Tasks: Define critical mission life support and exploration operations

Explore requirements imposed on mission ops by protection issues

Seek definition of base operations consistent with protection issues

Evening Dinner - Barbeque in Conference Center area

Saturday, June 23, 2001

7:00-8:15 a.m. Breakfast, informal at Dining Hall

8:30-noon Individual Working Group Meetings (continued)

Tasks: Continue exploration of mission requirements and opportunities consistent with planetary protection requirements Identify resources, solutions and recommendations Complete outline/planning for working group report Organize/Outline working group verbal report/presentation

Noon-1:00 p.m. $\quad$ Lunch, informal at Dining Hall

1:00-2:00 p.m. Plenary Session - Individual Working Group Reports and Discussions Group discussion on group reports

2:00-3:30 p.m. $\quad$ Plenary Session - Concluding Discussions and Formulation of Workshop Recommendations and Conclusions

3:30 p.m. Adjourn 


\section{Appendix B: Working Group Committee Members}

\section{WG1 - Protecting Mars and Science}

Penny Boston - Rapporteur

Fred Rainey - Recorder

Dale Andersen

Charles Barnes

Dean Eppler

Frank Grunthaner

Don Henninger

\section{WG2 - Protecting Human Health}

Robert Phillips - Rapporteur

John Charles - Recorder

Gary Martin

Brenda Ward

\section{WG3 - Protecting Earth}

Margaret Race - Rapporteur

Judy Allton - Recorder

John Batista

Kent Joosten

\section{WG4A - Operations I}

Mark Kliss - Rapporteur

Mark Lupisella - Recorder

Dale Andersen

John Battista

Penny Boston

Richard Fullerton

Don Henninger

Steven Hoffman

\section{WG4B - Operations II}

Brenda Ward - Rapporteur

Todd Stevens - Recorder

Judy Allton

Charles Barnes

John Charles

Dean Eppler

Frank Grunthaner

Kent Joosten

\author{
Mark Lupisella \\ Daniel Prieur \\ Perry Stabekis \\ Russell Vreeland \\ Diana Wall \\ Katie Harris - student \\ Melinda Miller - student
}

Mark Kliss

Brent Sherwood

Jeffrey Carlson - student

Gary Martin

Robert Phillips

Daniel Prieur

Margaret Race

Perry Stabekis (split time with WG4A and WG4B)

Albert Yen

Jeffrey Carlson - student

Katie Harris - student

Fred Rainey

Brent Sherwood

Perry Stabekis

Russell Vreeland

Robert Zimmerman

Lee Criswell - student

Howard Perko - student

Melinda Miller - student

\section{Others not assigned to specific working groups}

Marvin Criswell - Workshop organizing committee

Chris McKay - Workshop organizing committee

Marilee Rowe - Workshop support

John Rummel - Workshop convener 


\section{Appendix C: Workshop Participants List and Addresses}

\section{Invited Participants}

\section{Judith Allton}

Advanced Curation for Planetary Samples

Lockheed Martin/ Johnson Space Center

Mail Code C23

2400 NASA Road 1

Phone: 281-483-5766

Fax: 281-483-5347

E-mail: judith.h.allton1@jscc.nasa.gov

\section{Dale Andersen}

Department of Geography

McGill University

Burnside Hall

805 Sherbrooke Street West

Montreal, QC H3A 2K6

Canada

Phone: 650-604-5499

E-mail: andersen@gal.arc.nasa.gov

\section{Charles Barnes}

Advanced Human Support Technology Program

NASA Headquarters

Code UB

300 E Street SW

Washington, DC 20546

Phone: 202-358-2365

Fax: 202-358-4168

E-mail: cbarnes@hq.nasa.gov

\section{John Battista}

Department of Biological Sciences

626 Life Sciences Building

South Campus Drive

Louisiana State University

Baton Rouge, LA 70808

Phone: 225-388-2810

Fax: 225-388-2597

E-mail: jbattis@1su.edu

\author{
Penelope J. Boston \\ Director of Research \\ Complex Systems Research, Inc. \\ P.O. Box 11320 \\ Boulder, CO 80301 \\ Phone: 303-530-2661 \\ Fax: 303-581-9820 \\ E-mail:pboston@complex.org
}

\author{
John Charles \\ Mail Code SF2 \\ NASA - Lyndon B. Johnson Space Center \\ 2101 NASA Road 1 \\ Houston, TX 77058 \\ Phone: 281-483-7224 \\ Fax: 281-483-5773 \\ E-mail: john.b.charles@jsc.nasa.gov
}

\section{Dean Eppler}

Mail Code OZ4

NASA - Lyndon B. Johnson Space Center Houston, TX 77062

Phone: 281-244-8216

E-mail: dean.b.eppler1@,jsc.nasa.gov

\section{Frank Grunthaner}

Jet Propulsion Laboratory

MS 302-306

4800 Oak Grove Drive

Pasadena, CA 91109

Phone: 818-354-5564

Fax: 818-393-4540

E-mail: Frank.J.Grunthaner@jpl.nasa.gov

\section{Donald Henninger}

Mail Code EC

Building 7A, Room 234A

NASA - Johnson Space Center

2101 NASA Road 1

Houston, TX 77058-3696

Phone: 281-483-5034

Fax: 281-483-5060

E-mail: dhennin1@ems.jsc.nasa.gov 


\section{Stephen Hoffman}

SAIC

2200 Space Park Blvd, Suite 200

Houston, TX 77058

Phone: 281-483-9264

Fax: 281-244-7478

E-mail: stephen.j.hoffman1@j.jsc.nasa.gov

Kent Joosten

Exploration Analysis and Integration Office Mail Code EX 13

NASA - Lyndon B. Johnson Space Center 2102 NASA Road 1

Houston, TX 77058-3696

Phone: 281-483-4645

Fax: 281-224-7478

E-mail: kent.b.joosten@jsc.nasa.gov

\section{Mark Kliss}

Chief, Advanced Life Support Branch

MS 239-15

NASA Ames Research Center

Moffett Field, CA 94035-1000

Phone: 415-604-6246

Fax: 415-604-1092

E-mail: mkliss@mail.arc.nasa.gov

\section{Mark Lupisella}

NASA/Goddard Space Flight Center MS 584

Building 23, Room W207

Greenbelt Road

Greenbelt, MD 20771

Phone: 301-286-2918

Fax: 301-286-2325

E-mail: mark.lupisella@gsfc.nasa.gov

\section{Gary Martin}

Space Flight Office

Code MP

NASA Headquarters

Washington, DC 20546

Phone: 202-358-4470

Fax: 202-358-2818

E-mail: gmartin@hq.nasa.gov

\section{Robert Phillips}

Department of Psychology

110A Psychology Building

Colorado State University

Fort Collins, CO 80524-2202

Or

2 Windjammer Cove

Fort Collins: 970-484-1888

Phone (@CSU): 970-491-2983

E-mail: rwphil@lamar.colostate.edu

\section{Daniel Prieur}

University de Bretagne Occidentale

Institute Universitaire Europeen de la Mer

Place Nicolas Copernic

University of Brest

29280 Plouzane

France

Phone: 33-02-98-49-87-04

Fax: 33-02-98-49-87-05

E-Mail: Daniel.Prieur@univ-brest.fr

\section{Margaret Race}

1709 Greenhills Court

Lafayette, CA 94549

Phone: 925-947-1272

E-mail: mracemom@aol.com

\section{Fred Rainey}

Department of Biological Science

508 Life Sciences Building

Louisiana State University

Baton Rouge, LA 70808

Phone: 225-235-7160

E-mail: frainey@lsu.edu

\section{Brent Sherwood}

Senior Manager, Strategic Architectures/New Initiatives

Human Space Flight and Exploration

The Boeing Company

5301 Bolsa Avenue

MC H014-C443

Huntington Beach, CA 92647-2099

Phone: 714-372-9408

Fax: 714-372-2716

E-mail: brent.sherwood@boeing.com 
Pericles D. Stabekis

Windermere Group

525 School Street, SW, Suite 201

Washington, DC 20024

Phone: 202-484-8247

Fax: 202-484-8251

E-mail: pstabeks@hq.nasa.gov

\section{Todd Stevens}

1710 State Road

Mosier, OR 97040-9778

Phone: 541-478-0016

Fax: 541-478-0015

E-mail: tstevens@gorge.net

\section{Russell Vreeland}

Department of Biology

119 Schmucker Science Center

West Chester University

West Chester, PA 19320

Phone: 610-436-2479

Fax: 610-436-2183

E-mail: rvreeland@wcupa.edu

\section{Diana Wall}

Director, Natural Resource Ecology Laboratory

College of Natural Resources

Colorado State University

Fort Collins, CO 80523-1499

Phone: 970-491-2504

Fax: 970-491-1965

E-mail: diana@nrel.colostate.edu

\section{Brenda Ward}

Advanced Development Office

NASA - Lyndon B. Johnson Space Center

2101 NASA Road 1

Houston, TX 77058

Phone: 281-483-4657

Fax: 281-483-5800

E-mail: brenda.l.ward1@jsc.nasa.gov

\section{Albert Yen}

Jet Propulsion Laboratory

MS 183-501

4800 Oak Grove Drive

Pasadena, CA 91109

Phone: 818-354-7101

Fax: 818-393-5059

E-mail: albert.s.yen@jpl.nasa.gov

\section{Robert Zimmerman}

Synbiotek

265 Old Spanish Trail

Portola Valley, CA 94028

Phone: 650-851-2689

Fax: 650-851-3969

E-mail: rrzimmer@earthlink.net

\section{Organizing Participants}

Marvin E. Criswell

Workshop Co-Chairman \& Host

Department of Civil Engineering

Colorado State University

Fort Collins, CO 80523-1372

Phone: 970-491-5048

Fax: 970-491-7727

E-mail: mcriswel@engr.colostate.edu

Christopher P. McKay

Workshop Co-Chairman

Space Science Division

NASA-ARC

MS 245-3

Moffett Field, CA 94035

Phone: 650-604-6864

Fax: 650-604-6779

E-mail: mckay@arc.nasas.gov

John D. Rummel

Workshop Convener

Planetary Protection Officer

Code $\mathrm{S}$

NASA Headquarters

300 E Street SW

Washington, DC 20546

Phone: 202-358-0702

Fax: 202-358-3097

E-mail: jrummel@hq.nasa.gov

\section{Conference Support and Assistance}

\section{Marilee Rowe}

Department of Civil Engineering

Colorado State University

Fort Collins, CO 80523-1372

Phone: 970-491-5247

Fax: 970-491-6787

E-mail: mrowe@engr.colostate.edu 


\section{Student Participants}

\section{Jeffrey Carlson}

(Junior in Civil Engineering)

720 City Park, Apt C333

Fort Collins, CO 80521

Phone: 970-419-0095

E-mail: carlson@engr.colostate.edu

\section{Lee Criswell}

(Ph.D. Student in Environmental Health)

1536 Freedom Lane

Fort Collins, CO 80526-1707

Phone: 970-484-9566

E-mail: leecris@lamar.colostate.edu

\section{Katie Harris}

(Spring 2000 High school graduate, guest of Penny Boston)

\section{Melinda Miller}

(Sophomore in Civil Engineering)

820A West Laurel Street

Fort Collins, CO 80521

Phone: 970-472-1615

E-mail: mm946482@holly.colostate.edu

\section{Howard Perko}

(Ph.D. student in Civil Engineering)

727 Laporte Avenue

Fort Collins, CO 80521

Phone: 970-472-5668

E-mail: howie@engr.colostate.edu 


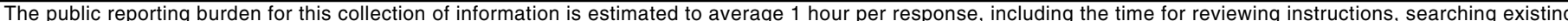
data sources, gathering and maintaining the data needed, and completing and reviewing the collection of information. Send comments regarding this burden estimate or any other aspect of this collection of information, including suggestions for reducing this burden, to Department of Defense, Washington Headquarters Services,

Directorate for information Operations and Reports (0704-0188), 1215 Jefferson Davis Highway, Suite 1204, Arlington, VA 22202-4302. Respondents should be aware that notwithstanding any other provision of law, no person shall be subject to any penalty for failing to comply with a collection of information if it does not display a currently valid OMB control number.

PLEASE DO NOT RETURN YOUR FORM TO THE ABOVE ADDRESS

\section{REPORT DATE (DD-MM-YYYY) 15-06-2005 \\ Conference Publication}

4. TITLE AND SUBTITLE

Planetary Protection Issues in the Human Exploration of Mars

\section{DATES COVERED (From - To)}

5a. CONTRACT NUMBER

SSX A63616

5b. GRANT NUMBER

5c. PROGRAM ELEMENT NUMBER
6. AUTHOR(S)

Criswell, Marvin E. ${ }^{1}$, Race, M. S. ${ }^{2}$, Rummel, J. D. ${ }^{3}$, and Baker, A. ${ }^{4}$

\section{5d. PROJECT NUMBER}

5e. TASK NUMBER

5f. WORK UNIT NUMBER

RTOP 344-42-02

8. PERFORMING ORGANIZATION REPORT NUMBER

${ }^{1}$ Colorado State University, Fort Collins, Colorado 80523

${ }^{2}$ SETI Institute, Mountain View, California 94043

${ }^{3}$ NASA Headquarters, Code SR, 300 E St. SW, Washington, D.C. 20546

${ }^{4}$ Technical Administrative Services (TAS), Littleton, Colorado

9. SPONSORING/MONITORING AGENCY NAME(S) AND ADDRESS(ES)

Ames Research Center

Moffett Field, CA 94035-1000 and

National Aeronautics and Space Administration

Washington, D.C. 20546-0001

REPORT NUMBER

NASA/CP-2005-213461

12. DISTRIBUTION/AVAILABILITY STATEMENT

Unclassified - Unlimited

Distribution: Nonstandard

Subject Category 51, 55, 88

Availability: NASA CASI (301) 621-0390

13. SUPPLEMENTARY NOTES

Point of Contact: Mark L. Fonda, Ames Research Center, MS245-1, Moffett Field, CA 94035-1000

(650) 604-5744

14. ABSTRACT

This workshop report, long delayed, is the first 21 st century contribution to what will likely be a series of reports examining the effects of human exploration on the overall scientific study of Mars. The considerations of human-associated microbial contamination were last studied in a 1990 workshop ("Planetary Protection Issues and Future Mars Missions," NASA CP-10086, 1991), but the timing of that workshop allowed neither a careful examination of the full range of issues, nor an appreciation for the Mars that has been revealed by the Mars Global Surveyor and Mars Pathfinder missions. Future workshops will also have the advantage of Mars Odyssey, the Mars Exploration Rover missions, and ESA's Mars Express, but the Pingree Park workshop reported here had both the NCR's (1992) concern that "Missions carrying humans to Mars will contaminate the planet" and over a decade of careful study of human exploration objectives to guide them-and to reconcile. A daunting challenge, and one that is not going to be simple (as the working title of this meeting, "When Ecologies Collide?" might suggest), it is clear that the planetary protection issues will have to be addressed to enable human explorers to safely and competently extend out knowledge about Mars, and its potential as a home for life-whether martian or human.

15. SUBJECT TERMS

Planetary protection, Human exploration, Mars

\begin{tabular}{|l|l|l|}
\hline \multicolumn{2}{|l|}{ 16. SECURITY CLASSIFICATION OF: } \\
\hline a. REPORT & b. ABSTRACT & c. THIS PAGE \\
Unclassified & Unclassified & Unclassified \\
\hline
\end{tabular}
ABSTRACT

Unclassified

\begin{tabular}{|c|c|}
$\begin{array}{c}\text { 18. } \begin{array}{l}\text { NUMBER } \\
\text { OF } \\
\text { PAGES }\end{array} \\
86\end{array}$ & $\begin{array}{l}\text { 19a. NAME OF RESPONSIBLE PERSON } \\
\text { Mark Fonda }\end{array}$ \\
\cline { 2 - 2 } & $\begin{array}{l}\text { 19b. } \begin{array}{l}\text { TELEPHONE (Include area code) } \\
(650) 604-5744\end{array} \\
\end{array}$
\end{tabular}

